\title{
Relativistic classical integrable tops and quantum R-matrices
}

\author{
A. Levin, ${ }^{a, b}$ M. Olshanetsky ${ }^{b, c}$ and A. Zotov ${ }^{d, b, c}$ \\ ${ }^{a}$ NRU HSE, Department of Mathematics, \\ Myasnitskaya str. 20, Moscow, 101000, Russia \\ ${ }^{b}$ ITEP, \\ B. Cheremushkinskaya str. 25, Moscow, 117218, Russia \\ ${ }^{c}$ MIPT, \\ Inststitutskii per. 9, Dolgoprudny, Moscow region, 141700, Russia \\ ${ }^{d}$ Steklov Mathematical Institute RAS, \\ Gubkina str. 8, Moscow, 119991, Russia \\ E-mail: alevin@hse.ru, olshanet@itep.ru, zotov@mi.ras.ru
}

ABSTRACT: We describe classical top-like integrable systems arising from the quantum exchange relations and corresponding Sklyanin algebras. The Lax operator is expressed in terms of the quantum non-dynamical $R$-matrix even at the classical level, where the Planck constant plays the role of the relativistic deformation parameter in the sense of Ruijsenaars and Schneider (RS). The integrable systems (relativistic tops) are described as multidimensional Euler tops, and the inertia tensors are written in terms of the quantum and classical $R$-matrices. A particular case of $\mathrm{gl}_{N}$ system is gauge equivalent to the $N$-particle RS model while a generic top is related to the spin generalization of the RS model. The simple relation between quantum $R$-matrices and classical Lax operators is exploited in two ways. In the elliptic case we use the Belavin's quantum $R$-matrix to describe the relativistic classical tops. Also by the passage to the noncommutative torus we study the large $N$ limit corresponding to the relativistic version of the nonlocal $2 \mathrm{~d}$ elliptic hydrodynamics. Conversely, in the rational case we obtain a new $\mathrm{gl}_{N}$ quantum rational non-dynamical $R$-matrix via the relativistic top, which we get in a different way - using the factorized form of the RS Lax operator and the classical Symplectic Hecke (gauge) transformation. In particular case of $\mathrm{gl}_{2}$ the quantum rational $R$-matrix is 11-vertex. It was previously found by Cherednik. At last, we describe the integrable spin chains and Gaudin models related to the obtained $R$-matrix.

Keywords: Integrable Equations in Physics, Quantum Groups, Gauge Symmetry

ARXIV EPRINT: 1405.7523 


\section{Contents}

1 Introduction 1

$\begin{array}{lll}2 & \text { Sklyanin algebras and classical integrable systems } & 7\end{array}$

$\begin{array}{lll}2.1 & \text { Quantum Sklyanin algebra } & 7\end{array}$

2.2 Classical limit 8

$\begin{array}{llr}2.3 & \text { Relativistic top } & 9\end{array}$

$\begin{array}{llr}2.4 & \text { Non-relativistic limit } & 10\end{array}$

$\begin{array}{lll}2.5 & \eta \text {-independent quadratic Poisson brackets } & 12\end{array}$

3 Relativistic rational top $\quad \mathbf{1 4}$

3.1 Factorized $L$-operators for classical Ruijsenaars-Schneider model $\quad 14$

$\begin{array}{lll}3.2 & \text { Lax pair } & 16\end{array}$

$\begin{array}{ll}3.3 & \text { Spin chains and Gaudin models } \\ \end{array}$

4 Quantum rational R-matrix $\quad 21$

5 Belavin's $\boldsymbol{R}$-matrix and elliptic models $\quad \mathbf{2 4}$

5.1 Sklyanin algebra and relativistic elliptic tops 24

$\begin{array}{lll}5.2 & \text { Poincaré invariance } & 27\end{array}$

5.3 Large $N$ limit: 2d elliptic hydrodynamics 29

6 Conclusion $\quad 32$

\section{Introduction}

We start with the quantum exchange relations $[40-45]$ for the quantum $\mathrm{gl}_{N}$-valued $L$ operators:

$$
R_{12}^{\hbar}(z-w) \hat{L}_{1}^{\eta}(z) \hat{L}_{2}^{\eta}(w)=\hat{L}_{2}^{\eta}(w) \hat{L}_{1}^{\eta}(z) R_{12}^{\hbar}(z-w)
$$

where the quantum non-dynamical $R$-matrix satisfies the quantum Yang-Baxter equation

$$
R_{12}^{\hbar}(z-w) R_{13}^{\hbar}(z) R_{23}^{\hbar}(w)=R_{23}^{\hbar}(w) R_{13}^{\hbar}(z) R_{12}^{\hbar}(z-w)
$$

and unitarity condition

$$
R_{12}^{\hbar}(z) R_{21}^{\hbar}(-z)=f^{\hbar}(z) 1 \otimes 1
$$

with some function $f^{\hbar}(z)$.

In this paper we consider a class of solutions of (1.1) and (1.2) having simple pole at $z=0$ and satisfying relation

$$
\hat{L}^{\eta}(z)=\operatorname{tr}_{2}\left(R_{12}^{\eta}(z) \hat{S}_{2}\right), \quad \hat{S}=\operatorname{Res}_{z=0} \hat{L}^{\eta}(z)
$$


where $\hat{S}$ is $\mathrm{gl}_{N}$-valued operator. Then (1.1) leads to (quadratic) Sklyanin algebra [72, 73] for $\hat{S}$ which we denote as $\mathcal{A}_{\hbar, \eta}^{\text {Skl }}$. Notice here that we use two parameters $\hbar$ and $\eta$ in (1.1) (it is customary to consider $\eta=\hbar$ ). In fact, one can even eliminate the $\eta$-dependence (see (1.20)) but we will see that it is useful to keep two free parameters from the very beginning.

In the classical limit $\hbar \rightarrow 0$ the matrix components of the residue $\hat{S}$ become $\mathbb{C}$-valued coordinates on the phase space of an integrable system described by the Lax matrix $L^{\eta}(z)$ (it coincides with $\hat{L}^{\eta}(z)$, where $\hat{S}$ is replaced with $\operatorname{gl}(N, \mathbb{C})$-valued $S$ )

$$
L^{\eta}(z)=\operatorname{tr}_{2}\left(R_{12}^{\eta}(z) S_{2}\right), \quad S=\operatorname{Res}_{z=0} L^{\eta}(z)
$$

and the standard quadratic $r$-matrix structure:

$$
\left\{L_{1}^{\eta}(z), L_{2}^{\eta}(w)\right\}=\left[L_{1}^{\eta}(z) L_{2}^{\eta}(w), r_{12}(z-w)\right]
$$

with the classical $r$-matrix $r_{12}(z)$. We call this type of models relativistic integrable tops because $\eta$ will be shown to play the role of the relativistic deformation parameter in the sense of Ruijsenaars and Schneider [69, 70]. The underlying Poincaré invariance is discussed in section 5.2.

Thus, when $\eta=\hbar$ we have simple relation (1.5) between the classical Lax operator and the quantum $R$-matrix, i.e. having quantum $R$-matrix we can define the classical integrable system. Write $R$-matrix in the standard $\mathrm{gl}_{N}$ basis $\left(\mathrm{E}_{i j}\right)_{a b}=\delta_{i a} \delta_{j b}$ as

$$
R_{12}^{\hbar}(z)=\sum_{i, j, k . l=1}^{N} R_{i j, k l}^{\hbar}(z) \mathrm{E}_{i j} \otimes \mathrm{E}_{k l} .
$$

Then it follows from (1.5) that

$$
L^{\eta}(z)=L^{\eta}(z, S)=\sum_{i, j, k, l=1}^{N} R_{i j, k l}^{\eta}(z) \mathrm{E}_{i j} S_{l k} .
$$

The latter leads to the converse statement, i.e. having the classical Lax matrix $L^{\eta}(z)$ we can find the quantum $R$-matrix as

$$
R_{12}^{\hbar}(z)=\frac{\partial L_{1}^{\hbar}(z)}{\partial S_{2}}=\sum_{k, l=1}^{N} \frac{\partial L^{\hbar}(z)}{\partial S_{l k}} \otimes \mathrm{E}_{k l}
$$

The purpose of the paper is twofold. The first one is to give description of the relativistic classical tops arising from the quantum $R$-matrices following (1.5). The second - is to derive new rational quantum $R$-matrix from the corresponding relativistic top via (1.9), which we obtain in a different way - by applying gauge transformation of Hecke type $[6,47-53,75]$ to the rational Ruijsenaars-Schneider (RS) model [69, 70]. 
1. Relativistic classical tops from quantum $\boldsymbol{R}$-matrices. Using local expansion of $L$-operator and $R$-matrix near $z=0$ we get equations of motion related to the Hamiltonian $\mathcal{S}_{0}=\operatorname{tr}(S)$ for the relativistic top in the form:

$$
\partial_{t_{0}} S=\left\{\mathcal{S}_{0}, S\right\} \stackrel{(1.5)-(1.6)}{=}\left[S, J^{\eta}(S)\right],
$$

where the inverse inertia tensor $J^{\eta}$ is the following linear functional:

$$
J^{\eta}(S)=\operatorname{tr}_{2}\left(\left(R_{12}^{\eta,(0)}-r_{12}^{(0)}\right) S_{2}\right)
$$

with $R_{12}^{\eta,(0)}$ and $r_{12}^{(0)}$ be zero terms in the expansions (2.3), (2.13) near $z=0$. See examples (3.36) and (1.23). These equations are presented in the Lax form

$$
\partial_{t_{0}} L^{\eta}(z)=\left\{\mathcal{S}_{0}, L^{\eta}(z)\right\} \stackrel{(1.5)-(1.6)}{=}\left[L^{\eta}(z), M(z)\right]
$$

with $M$-operator defined in terms of the classical $r$-matrix:

$$
M(z)=-\operatorname{tr}_{2}\left(r_{12}(z) S_{2}\right) .
$$

The latter $M$-operator appears to be equal (up to sign) to the non-relativistic limit of the Lax matrix $L^{\eta}(z)$ :

$$
L^{\eta}(z)=\eta^{-1} \frac{\mathcal{S}_{0}}{N} 1_{N \times N}+l(z)+\eta \mathcal{M}(z)+O\left(\eta^{2}\right), \quad l(z)=\operatorname{tr}_{2}\left(r_{12}(z) S_{2}\right)=-M(z) .
$$

Moreover, the next term in the expansion is the $M$-operator

$$
\partial_{t} l(z, S)=[l(z, S), \mathcal{M}(z, S)]
$$

of the non-relativistic top given by equation

$$
\partial_{t} S=[S, \mathrm{~J}(S)], \quad \mathrm{J}(S)=\mathcal{M}(0, S),
$$

where $\mathcal{M}(0)$ is the non-relativistic limit of $J^{\eta}(S)(1.11)$.

The model (1.16) is bihamiltonian. It means that it can be described by a pair of compatible Poisson structures. The first one (the Poisson-Lie) is generated by the linear $r$-matrix structure

$$
\left\{l_{1}(z), l_{2}(w)\right\}=\left[l_{1}(z)+l_{2}(w), r_{12}(z-w)\right],
$$

and the second - is by quadratic one

$$
\left\{\mathrm{L}_{1}(z), \mathrm{L}_{2}(w)\right\}=\left[\mathrm{L}_{1}(z) \mathrm{L}_{2}(w), r_{12}(z-w)\right]
$$

with

$$
\mathrm{L}(z, S)=\frac{\mathrm{s}_{0}}{N} 1+l(z, S)-\frac{\operatorname{tr} l(z, S)}{N} 1,
$$

where $\mathrm{s}_{0}$ is additional generator (of the classical Sklyanin algebra). In the elliptic case (corresponding to the Belavin-Drinfeld classical $r$-matrix [17]) this type of bihamiltonian structure for (1.18)-(1.19) was described in [37]. 
Thus, we have two quadratic algebras - (1.6) with $\eta$-dependent Lax operator (1.5), and (1.18) with $\eta$-independent Lax operator (1.19). Both algebras are described by the same $r$-matrix. Then it is natural to expect a relation between $L^{\eta}(z, S)$ and $\mathrm{L}(z, S)$. It can be written explicitly:

$$
L^{\eta}\left(z+\eta_{0}, \mathrm{~L}\left(-\eta_{0}, S\right)\right)=\frac{\operatorname{tr} L^{\eta}\left(z+\eta_{0}, S\right)}{\operatorname{tr} S} \mathrm{~L}(z, S)
$$

where $\mathrm{L}\left(-\eta_{0}, S\right)$ is inserted into $L^{\eta}(z, S)$ as the second argument, $\eta_{0}=\eta_{0}(\eta)$ is a zero of function $\operatorname{tr} L^{\eta}(z, S) / \operatorname{tr} S$. In notations of this paper $\eta_{0}=-\eta$ in the elliptic case and $\eta_{0}=-\eta / N$ in the rational one. Then the change of variables from the $\eta$-independent description (1.19) to the $\eta$-dependent description (1.5) can be written as

$$
S \rightarrow-\left(\eta_{0} / \eta\right) \mathrm{L}\left(-\eta_{0}, S\right)
$$

Relation (1.20) allows also to find the $M$-operator for (1.19) as

$$
\mathrm{M}(z, S)=-\left(\eta_{0} / \eta\right) J^{-\left(\eta / \eta_{0}\right) z}(\mathrm{~L}(z, S))
$$

The simplest example of the relativistic top is obtained in the elliptic case, where the quantum $R$-matrix is the Belavin's one [16]. In $\mathrm{gl}_{2}$ case it coincides with the Baxter's one. Then for $S=\sum_{a=0}^{3} S_{a} \sigma_{a}$, where $\sigma_{a}$ are the Pauli matrices $\left(\sigma_{0}=1\right)$

$$
\begin{aligned}
& J^{\eta}(S)=\sum_{a=0}^{3} J_{a}^{\eta} S_{a} \sigma_{a}, \quad J_{0}^{\eta}=E_{1}(\eta), \quad J_{\alpha}^{\eta}=E_{1}\left(\eta+\omega_{\alpha}\right)-E_{1}\left(\omega_{\alpha}\right), \quad \alpha=1,2,3, \\
& E_{1}(z)=\partial_{z} \log \vartheta(z \mid \tau), \quad \omega_{1}=\tau / 2, \quad \omega_{2}=(1+\tau) / 2, \quad \omega_{3}=1 / 2 .
\end{aligned}
$$

In the elliptic case we also consider the large $N$ limit to the elliptic hydrodynamics $[37,64]$ by passage to the noncommutative torus description:

$$
\partial_{t} S(x)=\operatorname{ad}_{J^{\eta}(S)(x)}^{*} S(x)=\left[S(x), J^{\eta}(S)(x)\right]_{\theta}
$$

where $[f(x), g(x)]_{\theta}=\theta^{-1}(f \star g-g \star f)$ is the Moyal bracket ( $\star$ is the Moyal product) and $J^{\eta}(S)$ is the pseudo-differential operator given in (5.60) (cf. (5.23)).

2. Quantum rational $\boldsymbol{R}$-matrix. We propose the factorized form for the rational Ruijsenaars-Schneider (RS) Lax matrix

$$
L^{\mathrm{RS}}(z)=g^{-1}(z) g(z+\eta) e^{P / c},
$$

where $c$ is the light speed, $P$ is a diagonal matrix of the RS particles momenta, and $g(z)$ is the matrix depending on the RS particles coordinates $q$. The latter was introduced in [6], where the non-relativistic rational top was constructed similarly starting from the rational Calogero-Moser (CM) model [19-22]. The transformation $g(z)$ is known for the quantum elliptic and trigonometric RS models [7, 36] (where the quantum IRF-Vertex 
correspondence was described). The rational one was mentioned in [6]. ${ }^{1}$ By performing the gauge transformation

$$
L^{\eta}(z)=g(z) L^{\mathrm{RS}}(z) g^{-1}(z)=g(z+\eta) e^{P / c} g^{-1}(z)
$$

and re-expressing $L^{\eta}(z)$ in terms of its residue we come to the relativistic rational top. It corresponds to some special values of the Casimir functions, while arbitrary values are related in the same way to the spin RS model [39] (see also [11]). The answer is given in section 3.2 .

Then using (1.5) we obtain rational unitary quantum R-matrix. In $\mathrm{gl}_{2}$ case it is the 11-vertex $R$-matrix

$$
R^{\hbar}(z)=\left(\begin{array}{cccc}
\hbar^{-1}+z^{-1} & 0 & 0 & 0 \\
-\hbar-z & \hbar^{-1} & z^{-1} & 0 \\
-\hbar-z & z^{-1} & \hbar^{-1} & 0 \\
-\hbar^{3}-2 z \hbar^{2}-2 \hbar z^{2}-z^{3} & \hbar+z \hbar+z \hbar^{-1}+z^{-1}
\end{array}\right)
$$

obtained previously in $[76] .{ }^{2}$ In section 4 we obtain $\mathrm{gl}_{N}$ generalization of (1.27). Introduction of $\epsilon$ parameter as

$$
R^{\hbar, \epsilon}(z)=\epsilon R^{\epsilon \hbar}(\epsilon z)
$$

allows to interpret it as deformation of the $\mathrm{XXX} R$-matrix

$$
\lim _{\epsilon \rightarrow 0} R^{\hbar, \epsilon}(z)=R^{\mathrm{xxx}}(z)=\frac{1}{\hbar} 1 \otimes 1+\frac{1}{z} P_{12}, \quad P_{12}=\sum_{i, j=1}^{N} \mathrm{E}_{i j} \otimes \mathrm{E}_{j i} .
$$

Notice that in this limit the relativistic top (1.10), (1.11) becomes free mechanical system in the sense that $L^{\eta}(z)=\eta^{-1} \mathcal{S}_{0} 1+z^{-1} S$, and equations of motion are trivial $\dot{S}=0$. Therefore, the parameter $\epsilon$ can be also treated as an alternative definition of the coupling constant.

The Lax matrix (1.26) (which is gauge equivalent to the RS model) emerge from explicit change of variables:

$$
\begin{aligned}
L^{\eta}(z) & =\operatorname{tr}_{2}\left(R_{12}^{\eta}(z) S_{2}\right) \\
S_{i j}(\mathbf{q}, \mathbf{p}) & =\sum_{m=1}^{N} \frac{\left(q_{m}+\eta\right)^{\varrho(i)} e^{p_{m} / c}}{\prod_{k \neq m}\left(q_{m}-q_{k}\right)}(-1)^{\varrho(j)} \sigma_{\varrho(j)}(\mathbf{q}),
\end{aligned}
$$

where $\varrho(i)=\delta_{i \leq N-1}(i-1)+\delta_{i N} N$ (see (3.2)), while $\sigma_{j}(q)$ are elementary symmetric functions (3.21)-(3.23). The case (1.30) corresponds to rank one matrix $S$ and to special values of the Casimir function $\operatorname{det} L^{\eta}(z)$ of Poisson brackets (1.6). In the quantum elliptic and trigonometric cases the (1.30)-type formulae for $\hat{S}=\hat{S}\left(\frac{\partial}{\partial \mathbf{q}}\right)$ can be found in [7, 36] (see also [39]).

\footnotetext{
${ }^{1}$ These gauge transformations underly the Symplectic Hecke Correspondence [47] (see also [48-51, 75]) for the classical integrable systems in the Hitchin approach.

${ }^{2}$ It can be also obtained [74] by applying special limiting procedure to the Baxter elliptic $R$-matrix [15].
} 
In general case $L^{\eta}(z)=\operatorname{tr}_{2}\left(R_{12}^{\eta}(z) S_{2}\right)$, where all $S_{i j}$ are independent variables. For non-relativistic models it was shown in [47] that the top models on the special coadjoint orbit are gauge equivalent to Calogero-Moser (CM) systems [19-22] while generic orbits correspond to their spin generalizations. In the same way, the generic relativistic top can be treated as alternative form of the spin RS model [39]. The gauge transformations used in (1.26) are of the same form as in non-relativistic case, where they play the role of modifications of the underlying Higgs bundles. Hence, we deal with the relativistic version of the Symplectic Hecke Correspondence. It allows us to obtain the non-dynamical quantum $R$-matrix instead of direct usage of the quantum IRF-Vertex Correspondence [65, 66]. In this respect, we realize the latter correspondence by means of the relativistic version of the classical (Symplectic Hecke) one. It is also interesting to mention that in view of (1.20), (1.21) we obtain the same form of equations (1.16) for the (spin) RS and (spin) CM models

Spin chains and Gaudin models related to the 11-vertex rational $R$-matrix (1.27) and its classical limit are obtained straightforwardly. As an example we get the $\mathrm{gl}_{2}$ Gaudin model Hamiltonians emerging from non-relativistic limit of the inhomogeneous chain:

$$
\begin{aligned}
h_{a} & =\sum_{c \neq a}^{n} \operatorname{tr}\left(\hat{S}^{a} l\left(z_{a}-z_{c}, \hat{S}^{c}\right)\right)=\sum_{c \neq a}^{n} \operatorname{tr}_{12}\left(r_{12}\left(z_{a}-z_{c}\right) \hat{S}_{1}^{a} \hat{S}_{2}^{c}\right)= \\
& =\sum_{c \neq a}^{n} \frac{\operatorname{tr}\left(\hat{S}^{a} \hat{S}^{c}\right)}{z_{a}-z_{c}}-\left(z_{a}-z_{c}\right)\left(\hat{S}_{12}^{a}\left(\hat{S}_{11}^{c}-\hat{S}_{22}^{c}\right)+\hat{S}_{12}^{c}\left(\hat{S}_{11}^{a}-\hat{S}_{22}^{a}\right)\right)-\left(z_{a}-z_{c}\right)^{3} \hat{S}_{12}^{a} \hat{S}_{12}^{c} .
\end{aligned}
$$

Notice that the first term corresponds to the standard rational (XXX) Gaudin Hamiltonians.

The 11-vertex model is defined by the quantum local Hamiltonian of the homogeneous periodic spin $(1 / 2)$ chain on $n$ sites is of the form:

$$
H^{\text {local }}=\sum_{k=1}^{n} P_{k, k+1}-\eta^{2} \mathrm{E}_{21}^{k} \otimes\left(\mathrm{E}_{11}^{k+1}-\mathrm{E}_{22}^{k+1}\right)-\eta^{2}\left(\mathrm{E}_{11}^{k}-\mathrm{E}_{22}^{k}\right) \otimes \mathrm{E}_{21}^{k+1}-\eta^{4} \mathrm{E}_{21}^{k} \otimes \mathrm{E}_{21}^{k+1}
$$

where $\mathrm{E}_{i j}^{n+1}=\mathrm{E}_{i j}^{1}$ (here we use the dual generators $\mathrm{E}_{i j}:\left(\mathrm{E}_{i j}\right)_{a b}=\delta_{i a} \delta_{j b}, S_{j i}=\operatorname{tr}\left(\mathrm{E}_{i j} \hat{S}\right)$ ). It is a deformation of the XXX spin chain described by the only first term in (1.32):

$$
H^{\mathrm{xxx}}=\sum_{k=1}^{n} P_{k, k+1}, \quad P_{k, k+1}=\mathrm{E}_{11}^{k} \otimes \mathrm{E}_{11}^{k+1}+\mathrm{E}_{12}^{k} \otimes \mathrm{E}_{21}^{k+1}+\mathrm{E}_{21}^{k} \otimes \mathrm{E}_{12}^{k+1}+\mathrm{E}_{22}^{k} \otimes \mathrm{E}_{22}^{k+1} .
$$

\section{Acknowledgments}

The work was supported by RFBR grants 12-02-00594 (A.L. and M.O.) and 12-01-00482 (A.Z.). The work of A.L. was partially supported by AG Laboratory GU-HSE, RF government grant, ag. 11 11.G34.31.0023. The work of A.Z. was partially supported by the D. Zimin's fund "Dynasty" and by the Program of RAS "Basic Problems of the Nonlinear Dynamics in Mathematical and Physical Sciences" П19. 


\section{Sklyanin algebras and classical integrable systems}

\subsection{Quantum Sklyanin algebra}

Let the quantum $L$-operator (1.1) has the following expansions in spectral parameter near $z=0$ :

$$
\hat{L}^{\eta}(z)=L^{\eta}(z, \hat{S})=\sum_{k=-1}^{\infty} z^{k} L^{\eta,(k)}(\hat{S})=\frac{1}{z} \hat{S}+L^{\eta,(0)}(\hat{S})+z L^{\eta,(1)}(\hat{S})+O\left(z^{2}\right),
$$

where the residue $\hat{S}$ is $\mathrm{gl}_{N^{-}}$valued operator and $L^{\eta,(k)}$ are linear functionals of $\hat{S}$, i.e.

$$
\hat{L}^{\eta}(z)=\sum_{a, b} \sum_{k=-1}^{\infty} z^{k}{\stackrel{k}{\mathcal{R}^{\eta}} a, b}_{a_{a}} \hat{S}_{b}, \quad L^{\eta,(k)}(\hat{S})=\sum_{a, b}{\stackrel{k}{\mathcal{R}^{\eta}}}_{a, b} T_{a} \hat{S}_{b}
$$

in some basis $\left\{T_{a}\right\}$ of $\mathrm{gl}_{N}$. The coefficients $\stackrel{k}{\mathcal{R}^{\eta}}$ a,b are functions of a free constant parameter $\eta$ which role is explained below. Due to (1.1) the matrix elements $\hat{S}_{b}$ satisfy quadratic relations of $\mathcal{A}_{\hbar, \eta}^{\mathrm{Skl}}$ (see $(2.7)$ ) such as Sklyanin algebra [72, 73] or its different extensions [23, $63,67,68]$. Notice that the representation space of operators $\hat{S}_{a}$ is not fixed yet.

Similarly to (2.1) and (2.2) let the $R$-matrix be of the form:

$$
\begin{aligned}
R_{12}^{\hbar}(z) & =\sum_{k=-1}^{\infty} z^{k} R_{12}^{\hbar,(k)}=\frac{1}{z} P_{12}+R_{12}^{\hbar,(0)}+z R_{12}^{\hbar,(1)}+O\left(z^{2}\right), \quad R_{12}^{\hbar,(k)} \in \mathrm{gl}_{N}{ }^{\otimes 2} \\
R_{12}^{\hbar,(k)} & =\sum_{a, b} \mathcal{R}_{a, b}^{k} T_{a} \otimes T_{-b}
\end{aligned}
$$

where the generators $T_{-b}$ are dual to $T_{b}: \operatorname{tr}\left(T_{a} T_{b}\right)=\delta_{a+b}$, and $R_{12}^{\hbar,(-1)}=P_{12}=\sum_{a} T_{a} \otimes T_{-a}$ is the permutation operator. Formulae (2.3) and (2.4) imply the following simple link between $L$-operator and $R$-matrix:

$$
\hat{L}^{\eta}(z)=\operatorname{tr}_{2}\left(R_{12}^{\eta}(z) \hat{S}_{2}\right) .
$$

It is important to mention that we deal with two constants $\hbar$ and $\eta$ (1.1). While $\hbar$ plays the role of the Planck constant, the parameter $\eta$ will be shown to describe relativistic deformation in the sense of Ruijsenaars.

Using notations of (2.1) and (2.3) it easy to write down the quadratic relations of $\mathcal{A}_{\hbar, \eta}^{\mathrm{Skl}}$. Indeed, consider residue of (1.2) at $w=0$ :

$$
R_{12}^{\hbar}(z) \hat{L}_{1}^{\eta}(z) \hat{S}_{2}=\hat{S}_{2} \hat{L}_{1}^{\eta}(z) R_{12}^{\hbar}(z)
$$

Expanding this equation near $z=0$ we get identity $P_{12} \hat{S}_{1} \hat{S}_{2}=\hat{S}_{2} \hat{S}_{1} P_{12}$ for $z^{-2}$ terms while the coefficients behind $z^{-1}$ give rise to the Sklyanin algebra: ${ }^{3}$

$$
\mathcal{A}_{\hbar, \eta}^{\mathrm{Skl}}: \quad P_{12} L^{\eta,(0)}(\hat{S})_{1} \hat{S}_{2}+R_{12}^{\hbar,(0)} \hat{S}_{1} \hat{S}_{2}=\hat{S}_{2} L^{\eta,(0)}(\hat{S})_{1} P_{12}+\hat{S}_{2} \hat{S}_{1} R_{12}^{\hbar,(0)} .
$$

\footnotetext{
${ }^{3}$ In his original paper $[72,73]$ Sklyanin used $\eta=\hbar$.
} 
A typical representative for (2.3)-type of solutions of the Yang-Baxter equation (1.2) is the Belavin's elliptic $R$-matrix. It is considered in section 5 .

It follows from (2.4) that the Sklyanin algebra $\mathcal{A}_{\hbar, \hbar}^{\text {Skl }}($ with $\eta=\hbar$ ) has finite-dimensional representation ${ }^{4}$

$$
\rho\left(\mathcal{A}_{\hbar, \hbar}^{\mathrm{Skl}}\right): \quad \rho\left(\hat{S}_{a}\right)=T_{-a} \in \mathrm{gl}_{N}
$$

Then

$$
R_{12}^{\hbar,(k)}=\rho\left(L^{\hbar,(k)}(\hat{S})\right) .
$$

With this definition the quantum Yang-Baxter equation (1.2) coincides with exchange relations (1.1) in representation (2.8).

\subsection{Classical limit}

Quantum $\boldsymbol{R}$-matrix. In the classical limit $\hbar \rightarrow 0$ the operators $\hat{S}_{a}$ become $\mathbb{C}$-valued coordinates on the phase space of an integrable system described by the Lax matrix $L^{\eta}(z, S)$. Notice that relation (2.5) remains intact at classical level, i.e.

$$
L^{\eta}(z, S)=\operatorname{tr}_{2}\left(R_{12}^{\eta}(z) S_{2}\right)=\sum_{a, b} \sum_{k=-1}^{\infty} z^{k}{\stackrel{k}{\mathcal{R}^{\eta}}}_{a, b} T_{a} S_{b} .
$$

Therefore, having the classical Lax matrix $L^{\eta}(z)$ of the described type we can compute the quantum $R$-matrix in the following way:

$$
R_{12}^{\hbar}(z)=\sum_{b} \frac{\partial L^{\hbar}(z, S)}{\partial S_{b}} \otimes T_{-b}
$$

We will use this formula in section 3 for derivation of the rational $R$-matrix.

Classical $r$-matrix. Let the quantum $R$-matrix has the following expansion in the Planck constant $\hbar$ :

$$
R_{12}^{\hbar}(z)=\frac{1}{\hbar} 1 \otimes 1+r_{12}(z)+\hbar r_{12}^{\prime}(z)+O\left(\hbar^{2}\right) \in \mathrm{gl}_{N}{ }^{\otimes 2},
$$

where

$$
r_{12}(z)=\frac{P_{12}}{z}+r_{12}^{(0)}+O(z), \quad r_{12}^{\prime}(z)=r_{12}^{\prime}(0)+O(z) .
$$

and $r_{12}^{(0)}$ comes from $R_{12}^{\hbar,(0)}$ in $(2.3)$ :

$$
R_{12}^{\hbar,(0)}=\frac{1}{\hbar} 1 \otimes 1+r_{12}^{(0)}+O(\hbar) .
$$

The term $r_{12}(z)$ is the classical $r$-matrix. It is skew-symmetric

$$
\begin{aligned}
& r_{12}(z)=-r_{21}(-z), \\
& { }^{k} \mathcal{R}^{0}{ }_{a, b}=(-1)^{k+1}{\stackrel{k}{\mathcal{R}^{0}}{ }_{b, a},}^{k} \quad \mathcal{R}^{0}=\lim _{\hbar \rightarrow 0}\left(\mathcal{R}^{\hbar}-\hbar^{-1} 1 \otimes 1\right)
\end{aligned}
$$

\footnotetext{
${ }^{4} \mathrm{In} \mathrm{gl}_{2}$ case the original Sklyanin algebra has simple representation in terms of the Pauli matrices $\hat{S}_{a}=\sigma_{-a}=\sigma_{a}, a=0,1,2,3$, with $\sigma_{0}=1_{2 \times 2}$, which are used as basis $\left\{T_{a}\right\}$.
} 
and satisfies the classical Yang-Baxter equation:

$$
\left[r_{12}(z-w), r_{13}(z)\right]+\left[r_{12}(z-w), r_{23}(w)\right]+\left[r_{13}(z), r_{23}(w)\right]=0 .
$$

The latter arises from (1.2) and (2.12). Similarly, by substituting (2.12) into (1.1) we come to quadratic Poisson structure

$$
\lim _{\hbar \rightarrow 0} \frac{1}{\hbar}\left[\hat{L}_{1}^{\eta}(z), \hat{L}_{2}^{\eta}(w)\right]:=\left\{L_{1}^{\eta}(z), L_{2}^{\eta}(w)\right\}=\left[L_{1}^{\eta}(z) L_{2}^{\eta}(w), r_{12}(z-w)\right],
$$

where $L^{\eta}(z)$ is the classical $L$-operator (the Lax matrix).

\subsection{Relativistic top}

Let us define the relativistic top as an integrable model described by the Lax matrix (2.10) and the $r$-matrix structure (2.18):

$$
\begin{aligned}
\left\{L_{1}^{\eta}(z), L_{2}^{\eta}(w)\right\} & =\left[L_{1}^{\eta}(z) L_{2}^{\eta}(w), r_{12}(z-w)\right] \\
L^{\eta}(z) & =\operatorname{tr}_{2}\left(R_{12}^{\eta}(z) S_{2}\right)=\frac{1}{z} S+L^{\eta,(0)}(S)+z L^{\eta,(1)}(S)+O\left(z^{2}\right) .
\end{aligned}
$$

We will see that equations of motion have the form of the integrable multidimensional Euler (or Euler-Arnold) top. On the other hand, it will be shown below that the parameter $\eta$ plays the same role as the relativistic deformation parameter in the Ruijsenaars-Schneider generalization of Calogero-Moser models. This is why we call these type of models relativistic tops. ${ }^{5}$

Classical Sklyanin algebra. The phase space is parameterized by $N^{2}$ coordinates $\left\{S_{a}\right\}$. It is equipped with the following quadratic Poisson structure:

$$
\mathcal{A}_{\hbar=0, \eta}^{\mathrm{Skl}}: \quad\left\{S_{1}, S_{2}\right\}=\left[S_{1} S_{2}, r_{12}^{(0)}\right]+\left[L^{\eta,(0)}(S)_{1} S_{2}, P_{12}\right],
$$

where $r_{12}^{(0)}$ is defined in $(2.13)$ and $L^{\eta,(0)}(S)$ in (2.1) and (2.19). The brackets (2.20) can be obtained both - from the quantum algebra (2.7) (by taking the classical limit (2.12)) or from (2.19) by computing residue at $w=0$

$$
\left\{L_{1}^{\eta}(z), S_{2}\right\}=\left[L_{1}^{\eta}(z) S_{2}, r_{12}(z)\right]
$$

and evaluating the coefficient in front of $z^{-1}$. The Poisson brackets are degenerated. In order to restrict it on a symplectic leaf we need to fix Casimir functions $C_{k}(S)$. They appear as coefficients in the expansion of $\operatorname{det} L^{\eta}(z)$ which is known to be central element for the Poisson brackets (2.18):

$$
\operatorname{det} L^{\eta}(z)=\sum_{k=-N}^{\infty} z^{k} C_{k}(S) .
$$

\footnotetext{
${ }^{5}$ Our approach is in agreement with the one considered in [24-26] for relativistic particles and rotators (in an external fields and on curved spaces), where the authors also used the term relativistic top. Our case corresponds to their free top.
} 
The number of independent Casimir functions (in general) equals $N$. They can be accumulated from coefficients in front of nonpositive powers of $z$ in (2.22) (others are dependent). The Hamiltonians (including the Casimir functions) can be computed from the expansion near $z=0$ of

$$
\begin{aligned}
\frac{1}{k} \operatorname{tr}\left(L^{\eta}(z)\right)^{k} & =\frac{1}{k} \operatorname{tr}_{0,1, \ldots, k}\left(R_{01}^{\eta}(z) \ldots R_{0 k}^{\eta}(z) S_{1} \ldots S_{k}\right)= \\
& =\frac{1}{z^{k}} H_{k, k}+\frac{1}{z^{k-1}} H_{k, k-1}+\ldots+H_{k, 0}+\ldots, \quad k=1 \ldots N
\end{aligned}
$$

or from the spectral curve

$$
\operatorname{det}\left(\lambda-L^{\eta}(z)\right)=0 .
$$

The number of Casimir functions $N$ should be subtracted from the number of independent Hamiltonians $(2.23) N(N+1) / 2$. This gives $N(N-1) / 2$ for the Hamiltonians only. It equals to the half of dimension of a general symplectic leaf. The Poisson commutativity of the Hamiltonians $H_{k, l}$ is guaranteed by (2.19). Therefore, the model is integrable in the Liouville-Arnold sense.

Equations of motion and Lax pair. The simplest Hamiltonian is given by

$$
\mathcal{S}_{0}:=H_{1,1}=\operatorname{tr}(S) .
$$

To get equations of motion let us compute the trace over the second component (in tensor product $\mathrm{gl}_{N}{ }^{\otimes 2}$ ) of (2.20). It leads to the top-like equations

$$
\partial_{t_{0}} S=\left\{\mathcal{S}_{0}, S\right\}=\left[S, J^{\eta}(S)\right],
$$

where the inverse inertia tensor $J^{\eta}$ is the following linear functional of $S$ :

$$
J^{\eta}: \quad S \rightarrow J^{\eta}(S)_{1}=-\operatorname{tr}_{2}\left(r_{12}^{(0)} S_{2}\right)+L^{\eta,(0)}(S)_{1} \stackrel{(2.10)}{=} \operatorname{tr}_{2}\left(\left(R_{12}^{\eta,(0)}-r_{12}^{(0)}\right) S_{2}\right) .
$$

In a similar way, by applying $\operatorname{tr}_{2}$ to the both parts of (2.21) we get equations of motion (2.26) in the Lax form:

$$
\partial_{t_{0}} L^{\eta}(z)=\left\{\mathcal{S}_{0}, L^{\eta}(z)\right\}=\left[L^{\eta}(z), M(z)\right],
$$

where the $M$-operator equals

$$
M_{1}(z)=-\operatorname{tr}_{2}\left(r_{12}(z) S_{2}\right) .
$$

Notice that it is independent of $\eta$. As we will see below the $M$-operator in this description coincides with non-relativistic Lax matrix.

\subsection{Non-relativistic limit}

The non-relativistic limit $\eta \rightarrow 0$ is similar to the classical one due (2.10). It follows from (2.12) and (2.10) that

$$
\begin{aligned}
L^{\eta}(z) & =\frac{\mathcal{S}_{0}}{N \eta} 1_{N \times N}+\operatorname{tr}_{2}\left(r_{12}(z) S_{2}\right)+\eta \operatorname{tr}_{2}\left(r_{12}^{\prime}(z) S_{2}\right)+O\left(\eta^{2}\right), \\
L^{\eta,(0)} & =\frac{\mathcal{S}_{0}}{N \eta} 1_{N \times N}+\operatorname{tr}_{2}\left(r_{12}^{(0)} S_{2}\right)+\eta \operatorname{tr}_{2}\left(r_{12}^{\prime}(0) S_{2}\right)+O\left(\eta^{2}\right) .
\end{aligned}
$$


Plugging (2.30) and (2.31) into (2.19) and (2.20) respectively we get

$$
\left\{L_{1}^{\eta}(z), L_{2}^{\eta}(w)\right\}=\frac{\mathcal{S}_{0}}{N \eta}\left[l_{1}(z)+l_{2}(w), r_{12}(z-w)\right]+\left[l_{1}(z) l_{2}(w), r_{12}(z-w)\right]+O(\eta),
$$

where

$$
\begin{aligned}
l_{1}(z) & :=\operatorname{tr}_{2}\left(r_{12}(z) S_{2}\right), \\
l(z) & =\frac{1}{z} S+l^{(0)}(S)+z l^{(1)}(S)+O(z), \quad l^{(0)}(S)_{1}=\operatorname{tr}_{2}\left(r_{12}^{(0)} S_{2}\right) .
\end{aligned}
$$

and

$$
\left\{S_{1}, S_{2}\right\}=\frac{\mathcal{S}_{0}}{N \eta}\left[S_{2}, P_{12}\right]+\left[S_{1} S_{2}, r_{12}^{(0)}\right]+\left[\operatorname{tr}_{3}\left(r_{13}^{(0)} S_{3}\right) S_{2}, P_{12}\right]+O(\eta)
$$

where $\operatorname{tr}_{3}\left(r_{13}^{(0)} S_{3}\right)$ is just $l^{(0)}(S)_{1}$ as in $(2.34)$.

When $\eta \rightarrow 0$ the leading term in (2.35) is the linear Poisson-Lie structure on $\mathrm{gl}_{N}^{*}$ Lie coalgebra. The generator $\mathcal{S}_{0}=\operatorname{tr} S$ is the Casimir function of the latter brackets. Let us fix it as $\mathcal{S}_{0}=N$ and set

$$
\left\{S_{1}, S_{2}\right\}_{\text {Lie }}:=\lim _{\eta \rightarrow 0} \eta\left\{S_{1}, S_{2}\right\}
$$

Then

$$
\left\{S_{1}, S_{2}\right\}_{\text {Lie }}=\left[S_{2}, P_{12}\right] .
$$

In the same way the linear $r$-matrix structure is obtained at the level of Lax matrices:

$$
\left\{l_{1}(z), l_{2}(w)\right\}_{\text {Lie }}=\left[l_{1}(z)+l_{2}(w), r_{12}(z-w)\right] .
$$

Non-relativistic top. We will refer to an integrable model described by the Lax matrix (2.33) and the Poisson structure (2.37), (2.38) as the non-relativistic top. The phase space is the coadjoint orbit of $\mathrm{GL}_{N}$ Lie group. It is equipped with the linear Poisson-Lie structure on $\mathrm{gl}_{N}^{*}$. For example, using the standard basis of matrices $\left(\mathrm{E}_{i j}\right)_{a b}=\delta_{i a} \delta_{j b}(2.37)$ acquires the from $\left\{S_{i j}, S_{k l}\right\}=\delta_{i l} S_{k j}-\delta_{k j} S_{i l}$. A general symplectic leaf is obtained by fixation of eigenvalues of $S$ or the Casimir functions $c_{k}=\frac{1}{k} \operatorname{tr}\left(S^{k}\right), k=1 \ldots N$. The Hamiltonians appear as in (2.23):

$$
\begin{aligned}
\frac{1}{k} \operatorname{tr}(l(z))^{k} & =\frac{1}{k} \operatorname{tr}_{0,1, \ldots, k}\left(r_{01}(z) \ldots r_{0 k}(z) S_{1} \ldots S_{k}\right)= \\
& =\frac{1}{z^{k}} h_{k, k}+\frac{1}{z^{k-1}} h_{k, k-1}+\ldots+h_{k, 0}+\ldots, \quad k=1 \ldots N .
\end{aligned}
$$

It is easy to see that $h_{k, k}=c_{k}$. The Poisson commutativity of the Hamiltonians $h_{k, l}$ is guaranteed by (2.38). The $M$-operators corresponding to the Hamiltonians $h_{k, l}$ are evaluated by expansion of $-\operatorname{tr}_{2}\left(r_{12}(z-w) l_{2}^{k-1}(w)\right)$ (see [14]). An alternative way is given in $(2.54)-(2.56)$.

Notice that the Poisson brackets (2.37) follows from (2.38) and local expansion from (2.34). To get (2.37) one should substitute expansion (2.34) into (2.38) and compute the residue at $w=0$ and then at $z=0$. 
Similarly to (2.10), we have a simple link between the Lax matrix and classical $r$-matrix given by (2.33). Substitution of (2.33) into (2.38) gives rise to the classical Yang-Baxter equation (2.17) (it follows from the Jacobi identity for the Poisson brackets (2.38) as well). By analogy with (2.11) we have

$$
r_{12}(z)=\sum_{b} \frac{\partial l(z)}{\partial S_{b}} \otimes T_{-b}
$$

This relation was used in [6] for computation of the rational classical $r$-matrix.

\section{5 $\eta$-independent quadratic Poisson brackets}

Let us consider another limit of brackets (2.32) and (2.35). Set

$$
\mathcal{S}_{0}=\eta \mathrm{s}_{0}
$$

With this rescaling $\mathcal{S}_{0} \rightarrow 0$ when $\eta \rightarrow 0$. Then the residue $S$ becomes traceless, i.e.

$$
\begin{aligned}
S & \stackrel{\eta \rightarrow 0}{\longrightarrow} \bar{S}=S-\frac{1}{N} \operatorname{tr}(S) 1_{N \times N}, \\
l(z) & \stackrel{\eta \rightarrow 0}{\longrightarrow} \bar{l}(z)=l(z)-\frac{1}{N z} \operatorname{tr}(S) 1_{N \times N}=\frac{1}{z} \bar{S}+l^{(0)}(S)+O(z) .
\end{aligned}
$$

Applying this limit to $(2.32)$ we get

$$
\left\{\mathrm{L}_{1}(z), \mathrm{L}_{2}(w)\right\}=\left[\mathrm{L}_{1}(z) \mathrm{L}_{2}(w), r_{12}(z-w)\right],
$$

where the Lax matrix

$$
\mathrm{L}(z):=\frac{\mathrm{s}_{0}}{N} 1+\bar{l}(z)
$$

The Poisson brackets (2.35) acquire the following form in the limit:

$$
\mathcal{A}_{\hbar=0, \eta=0}^{\mathrm{Skl}}: \quad\left\{\bar{S}_{1}, \bar{S}_{2}\right\}=\frac{\mathrm{s}_{0}}{N}\left[\bar{S}_{2}, P_{12}\right]+\left[\bar{S}_{1} \bar{S}_{2}, r_{12}^{(0)}\right]+\left[\operatorname{tr}_{3}\left(r_{13}^{(0)} \bar{S}_{3}\right) \bar{S}_{2}, P_{12}\right] .
$$

The missing brackets $\left\{\bar{S}, \mathrm{~s}_{0}\right\}$ can be found by taking the limit in (2.26). Plugging (2.41) into (2.26), (2.27) we get:

$$
\left\{\mathrm{s}_{0}, \bar{S}\right\}=\lim _{\eta \rightarrow 0} \frac{\left[S, J^{\eta}(S)\right]}{\eta}=[\bar{S}, \mathrm{~J}(\bar{S})]
$$

where the inverse inertia tensor $\mathrm{J}$ is defined as

$$
\mathrm{J}: \quad \bar{S}_{1} \rightarrow \mathrm{J}(\bar{S})_{1}=\operatorname{tr}_{2}\left(r_{12}^{\prime}(0) \bar{S}_{2}\right)
$$

with $r_{12}^{\prime}(0)$ from (2.31). Equations (2.47), (2.48) also play the role of equations of motion generated by the Hamiltonian $\mathrm{s}_{0}$ :

$$
\partial_{\mathrm{t}_{0}} \bar{S}=\left\{\mathrm{s}_{0}, \bar{S}\right\}=[\bar{S}, \mathrm{~J}(\bar{S})],
$$

The corresponding Lax equation can be obtained in two ways. The first one [14] — is by taking $\operatorname{tr}_{2}$ in (2.44). This yields

$$
\partial_{\mathrm{t}_{0}} \mathrm{~L}(z)=\left\{\mathrm{s}_{0}, \mathrm{~L}(z)\right\}=[\mathrm{L}(z), \mathrm{M}(z)]
$$


where

$$
\mathrm{M}_{1}(z)=-\operatorname{tr}_{2}\left(r_{12}(z-w) \mathrm{L}_{2}(w)\right) .
$$

The latter matrix should be $w$-independent up to some element from the kernel of $\operatorname{ad}_{\mathrm{L}(z)}$. Alternatively, one can consider the limit of $(2.28):\left\{\mathcal{S}_{0}, L^{\eta}(z)\right\}=\left[L^{\eta}(z), M(z)\right]$. Notice again that $M(z)$ given by $(2.29)$ is $\eta$-independent. Moreover, it coincides with $\mathrm{L}(z)$ up to sign and some scalar - element from $\operatorname{Ker}\left(\operatorname{ad}_{\mathrm{L}(z)}\right)$, i.e.

$$
\left\{\mathcal{S}_{0}, L^{\eta}(z)\right\}=\left[L^{\eta}(z), M(z)\right]=-\left[L^{\eta}(z), \mathrm{L}(z)\right] .
$$

Then, substituting rescaling (2.41) and using expansion (2.30) we get

$$
\partial_{\mathrm{t}_{0}} \mathrm{~L}(z)=\left\{\mathrm{s}_{0}, \mathrm{~L}(z)\right\}=\lim _{\eta \rightarrow 0} \frac{\left[\mathrm{L}(z), L^{\eta}(z)\right]}{\eta}=[\mathrm{L}(z), \mathcal{M}(z)],
$$

where

$$
\mathcal{M}(z)=\operatorname{tr}_{2}\left(r_{12}^{\prime}(z) \bar{S}_{2}\right) .
$$

with $r_{12}^{\prime}(z)$ defined in (2.30). Thus, the roles of $L$ and $M$-operators are interchanged while taking the limit. In addition, $r_{12}^{\prime}(z)$ has no singularities at $z=0$. Then

$$
\mathcal{M}(0)=\operatorname{tr}_{2}\left(r_{12}^{\prime}(0) \bar{S}_{2}\right) \stackrel{(2.48)}{=} \mathrm{J}(\bar{S}) .
$$

Finally, we see that the expansion (2.30) of $L^{\eta}(z)$ in $\eta$ provides $M$-operators for both $\eta$-dependent and $\eta$-independent descriptions:

$$
L^{\eta}(z)=\eta^{-1} \mathcal{S}_{0} / N-M(z)+\eta \mathcal{M}(z)+O\left(\eta^{2}\right) .
$$

Notice also that the $M$-operator (2.54) is also valid for the linear $r$-matrix structure (2.38) since the Lax pairs for the linear and quadratic ( $\eta$-independent) $r$-matrix structures are the same (up to scalar terms). The formulae obtained in this section can be considered as an extension of [14] for the class of integrable systems under consideration.

Relation between $\mathcal{A}_{\eta \neq 0}^{\mathrm{skl}}$ and $\mathcal{A}_{\eta=0}^{\mathrm{Skl}}$. We have two different description of the classical Sklyanin algebras (and related integrable models). In the first one the quadratic Poisson structure $(2.20) \mathcal{A}_{\hbar=0, \eta}^{\text {Skl }}$ is $\eta$-dependent. The second $\mathcal{A}_{\hbar=0, \eta=0}^{\text {Skl }}(2.46),(2.47)-$ is $\eta$-independent. The same happens at quantum level. One can quantize the Lax matrix $(2.45)$ as

$$
\hat{\mathrm{L}}(z):=\frac{1}{N} \hat{\mathrm{s}}_{0}+\operatorname{tr}_{2}\left(r_{12}(z) \hat{\bar{S}}_{2}\right) .
$$

Then the exchange relations (1.1) gives $\eta$-independent quadratic algebra $\mathcal{A}_{\hbar, \eta=0}^{\text {Skl }}$.

Algebras $\mathcal{A}_{\eta \neq 0}^{\mathrm{Skl}}$ and $\mathcal{A}_{\eta=0}^{\mathrm{Skl}}$ are related. The relation is easy to demonstrate explicitly in the elliptic case (see section 5). The idea is the following. There exists a linear functional $\varphi^{\eta}$ on $\mathrm{gl}_{N}$ (depending on the boundary conditions) such that (2.5) and (2.57) are related as follows:

$$
g^{\eta}(z) e^{\eta_{0} \partial_{z}} \hat{L}\left(z, \varphi^{\eta}(\hat{S})\right)=\hat{\mathrm{L}}(z, \hat{S}),
$$


where $g^{\eta}(z)$ is some function with a simple zero at $z=-\eta_{0}$ and simple pole at $z=0$. Finally, the relation can be written as

$$
L^{\eta}\left(z+\eta_{0}, \mathrm{~L}\left(-\eta_{0}, S\right)\right)=\frac{\operatorname{tr} L^{\eta}\left(z+\eta_{0}, S\right)}{\operatorname{tr} S} \mathrm{~L}(z, S) .
$$

It holds true in the rational case as well. We may use this relation to get explicit change of variables from the $\eta$-independent description (2.45) to the $\eta$-dependent (2.19):

$$
S \rightarrow c \mathrm{~L}(c \eta, S), \quad c=-\frac{\eta_{0}}{\eta} .
$$

The coefficient $-\eta_{0} / \eta$ is chosen in order to have $\operatorname{Res}_{\eta=0}\left(-\frac{\eta_{0}}{\eta} \mathrm{L}\left(-\eta_{0}, S\right)\right)=\operatorname{Res}_{z=0}(z, S)$. It is interesting to note that plugging this change of variables to the equations of motion $(2.26)$ gives

$$
\partial_{t} \mathrm{~L}(c \eta, S)=\left[\mathrm{L}(c \eta, S), c J^{\eta}(\mathrm{L}(c \eta, S))\right],
$$

i.e. the Lax equations (2.50), where $c \eta$ plays the role of the spectral parameter. Hence, we get an alternative definition for the $M$-operator

$$
\mathrm{M}(z, S)=c J^{z / c}(\mathrm{~L}(z, S)) .
$$

\section{Relativistic rational top}

In this section we obtain explicit answer for the Lax pair of the relativistic top. As it was already mentioned this model is a top-like form of the spin Ruijsenaars-Schneider (RS) model. To get the answer we represent the Lax matrix of RS model in the factorized form (3.6) which is convenient for the gauge transformation. The dynamical variables of the top are the components of the residue (3.18), (3.26) of the gauge transformed RS Lax matrix (3.16). We express the gauge transformed $L$-operator in terms of its residue. This gives the correct answer for generic top since it is independent of the Casimir functions values.

\subsection{Factorized $L$-operators for classical Ruijsenaars-Schneider model}

In this paragraph we propose factorized forms of $L$-operators for the rational RS model [69, 70].

Following [6] for the set of variables $\left\{q_{j}\right\}, j=1 \ldots N$ such that $\sum_{j=1}^{N} q_{j}=0$ let us introduce the matrix

$$
\Xi_{i j}(\mathbf{q}, z):=\left(z+q_{j}\right)^{\varrho(i)}, \quad i, j=1 \ldots N
$$

where

$$
\varrho(i)=\left\{\begin{array}{l}
i-1 \text { for } 1 \leq i \leq N-1, \\
i \quad \text { for } i=N .
\end{array} \quad \varrho^{-1}(i)=\left\{\begin{array}{l}
i+1 \text { for } 0 \leq i \leq N-2, \\
i \quad \text { for } i=N .
\end{array}\right.\right.
$$


It has the property

$$
\operatorname{det} \Xi(\mathbf{q}, z)=N z \prod_{1 \geq i>j \geq N}\left(q_{i}-q_{j}\right)
$$

i.e. the matrix is degenerated at $z=0$. It can be also treated as the rational analogue of the modification of bundles over elliptic curves used in [47] for the description of the elliptic top.

Rational $\mathrm{sl}_{N}$ RS model with spectral parameter is defined by the following Lax matrix:

$$
L_{i j}^{\mathrm{RS}}(z, \eta)=\eta\left(\frac{1}{q_{i}-q_{j}+\eta}-\frac{1}{N z}\right) e^{p_{j} / c} \prod_{k \neq j}^{N} \frac{q_{j}-q_{k}-\eta}{q_{j}-q_{k}}
$$

where

$$
\sum_{k=1}^{N} q_{k}=\sum_{k=1}^{N} p_{k}=0
$$

The classical $r$-matrix structure was found in $[12,13]$.

Proposition 3.1 The Lax matrix (3.4) can be written in the following form: ${ }^{6}$

$$
L^{R S}(z, \eta)=D_{0}(\mathbf{q}) \Xi^{-1}(\mathbf{q}, z) \Xi(\mathbf{q}, z-\eta) D_{0}^{-1}(\mathbf{q}) e^{P / c},
$$

where $D_{0}(\mathbf{q})$ is diagonal matrix $\left(D_{0}\right)_{i j}=\delta_{i j} \prod_{k \neq i}^{N}\left(q_{i}-q_{k}\right)$.

Let us also write the similar answer for ${ }^{7}$

Rational $\mathrm{sl}_{N}$ RS model without spectral parameter. The Lax matrix

$$
L_{i j}^{\mathrm{RS}}=\frac{\eta e^{p_{j} / c}}{q_{i}-q_{j}+\eta} \prod_{k \neq j}^{N} \frac{q_{j}-q_{k}-\eta}{q_{j}-q_{k}} .
$$

is represented in the form:

$$
\begin{aligned}
L^{\mathrm{RS}} & =D_{0}(\mathbf{q}) V^{-1}(\mathbf{q}, z) V(\mathbf{q}, z-\eta) D_{0}^{-1}(\mathbf{q}) e^{P / c}= \\
& =D_{0}(\mathbf{q}) V^{-1}(\mathbf{q}, z) C_{-\eta} V(\mathbf{q}, z) D_{0}^{-1}(\mathbf{q}) e^{P / c},
\end{aligned}
$$

where

$$
V_{i j}(\mathbf{q}, z):=\left(z+q_{j}\right)^{i-1}, \quad i, j=1 \ldots N
$$

and

$$
\left(C_{\lambda}\right)_{i j}=\left\{\begin{array}{l}
\frac{(i-1) ! \lambda^{i-j}}{(j-1) !(i-j) !}, j \leq i \\
0, \quad j>i
\end{array}\right.
$$

${ }^{6}$ The prove is direct. See formulae in section 3.2 and appendix in [6].

${ }^{7} \mathrm{It}$ is not used in the subsequent sections. 
It easy to verify that

$$
C_{\lambda}=\exp \left(\lambda C_{0}\right), \quad\left(C_{0}\right)_{i j}= \begin{cases}j, & i=j+1, i=2, \ldots, N \\ 0, & \text { otherwise }\end{cases}
$$

and $\partial_{z} V=C_{0} V$. The limit to Calogero-Moser model is obtained as follows:

$$
L_{i j}^{\mathrm{CM}}=\left.\lim _{c \rightarrow \infty} c L_{i j}^{\mathrm{RS}}\right|_{\eta=\nu / c}-\delta_{i j}=P-\nu D_{0} V^{-1} C_{0} V D_{0}^{-1} .
$$

Notice that we can also define the Lax matrix as

$$
L_{i j}^{\prime \mathrm{RS}}=\frac{\eta e^{p_{j} / c}}{q_{i}-q_{j}+\eta} \prod_{k \neq j}^{N} \frac{q_{j}-q_{k}+\eta}{q_{j}-q_{k}} .
$$

It differs from (3.7) by the canonical map

$$
e^{p_{j} / c} \longrightarrow e^{p_{j} / c} \prod_{k \neq j}\left(\frac{q_{j}-q_{k}+\xi}{q_{j}-q_{k}-\xi}\right)^{a}
$$

with $a=1$ and $\xi=\eta$. Then

$$
\begin{aligned}
L^{\prime \mathrm{RS}} & =D_{\eta}^{-1}(\mathbf{q})\left(V^{T}\right)(\mathbf{q}, z+\eta)\left(V^{T}\right)^{-1}(\mathbf{q}, z) D_{\eta}(\mathbf{q}) e^{P / c}= \\
& =D_{\eta}^{-1}(\mathbf{q})\left(V^{T}\right)(\mathbf{q}, z) C_{\eta}^{T}\left(V^{T}\right)^{-1}(\mathbf{q}, z) D_{\eta}(\mathbf{q}) e^{P / c}, \quad\left(D_{\lambda}\right)_{i j}=\delta_{i j} \prod_{k \neq i}^{N}\left(q_{i}-q_{k}+\lambda\right) .
\end{aligned}
$$

Let us mention that the transformation (3.9) was used in the classical [30] and the quantum [27-29] IRF-Vertex transformations. In this way the (Jordanian) $R$-matrices of the Cremmer-Gervais type were obtained.

\subsection{Lax pair}

Apply the gauge transformation $g(z)=\Xi(\mathbf{q}, z) D_{0}^{-1}(\mathbf{q})$ to the RS Lax matrix (3.4), (3.6) with $\eta:=-\eta$. Then it follows from (3.6) that

$$
\begin{aligned}
\tilde{L}^{\eta, c}(z, \mathbf{q}, \mathbf{p}): & :=\Xi(\mathbf{q}, z) D_{0}^{-1}(\mathbf{q}) L_{i j}^{\mathrm{RS}}(z,-\eta) D_{0}(\mathbf{q}) \Xi^{-1}(\mathbf{q}, z)= \\
& =\Xi(\mathbf{q}, z+\eta) e^{P / c} \Xi^{-1}(\mathbf{q}, z) .
\end{aligned}
$$

Let $\sum_{j=1}^{N} p_{j}=0$. Then from (3.3) it follows that

$$
\operatorname{det} \tilde{L}^{\eta, c}(z, \mathbf{q}, \mathbf{p})=\frac{z+\eta}{z} .
$$

Our purpose now is to express this matrix in terms of its residue at $z=0$. Set

$$
S=N \operatorname{Res}_{z=0} \tilde{L}^{\eta, c}(z, \mathbf{q}, \mathbf{p})=\frac{1}{\prod_{1 \geq i>j \geq N}\left(q_{i}-q_{j}\right)} \Xi(\mathbf{q}, \eta) e^{P / c} \operatorname{adj}(\Xi(\mathbf{q}, 0)),
$$


where the adjugate is transpose of the cofactor matrix. To find matrix components we need the inverse of $\Xi$ :

$$
\Xi_{k j}^{-1}(\mathbf{x})=(-1)^{\varrho(j)} \frac{\sigma_{\varrho(j)}(\mathbf{x})}{\left(\sum_{s=1}^{N} x_{s}\right) \prod_{s \neq k}^{N}\left(x_{k}-x_{s}\right)}-(-1)^{\varrho(j)} \frac{\stackrel{k}{\sigma}_{\varrho(j)}(\mathbf{x})}{\prod_{s \neq k}^{N}\left(x_{k}-x_{s}\right)},
$$

where $x_{j}=q_{j}+z$. Expansion in powers of $z$ gives

$$
\begin{aligned}
& \Xi_{m j}^{-1}(z, \mathbf{q})=\frac{1}{N z} \frac{(-1)^{\varrho(j)}}{\prod_{r \neq m}^{N}\left(q_{m}-q_{r}\right)}\left(\sigma_{\varrho(j)}(\mathbf{q})+\sum_{s=1}^{N-j} z^{s}\left[\sigma_{s+j-1}(\mathbf{q})\left(\begin{array}{c}
s+j-1 \\
j-1
\end{array}\right)\right.\right. \\
& \left.\left.-N \stackrel{m}{\sigma}_{s+j-2}(\mathbf{q})\left(\begin{array}{c}
s+j-2 \\
j-1
\end{array}\right)\right]-(N-j) z^{N-j+1}{\underset{\sigma}{N-1}}_{N}^{m}(\mathbf{q})\left(\begin{array}{c}
N \\
j-1
\end{array}\right)\right) .
\end{aligned}
$$

In (3.19) and (3.20) the elementary symmetric functions are used:

$$
\prod_{k=1}^{N}\left(\zeta-x_{k}\right)=\sum_{k=0}^{N}(-1)^{k} \zeta^{k} \sigma_{k}(\mathbf{x})
$$

or

$$
\sigma_{N-d}(\mathbf{x})=(-1)^{N} \sum_{1 \leq i_{1}<i_{2} \ldots<i_{d} \leq N} x_{i_{1}} x_{i_{2}} \ldots x_{i_{d}}, \quad d=0, \ldots, N
$$

and their derivatives

$$
-\prod_{m \neq k}^{N}\left(\zeta-x_{m}\right)=\sum_{s=0}^{N-1}(-1)^{s} \zeta^{s} \stackrel{k}{\sigma}_{s}(\mathbf{x})
$$

These functions satisfy the following set of identities:

$$
\begin{aligned}
& \stackrel{m}{\sigma}_{j}(\mathbf{x})=\sum_{c=0}^{N-j-1}\left(-x_{m}\right)^{c} \sigma_{j+1+c}(\mathbf{x}), \\
& \stackrel{m}{\sigma}_{j}(\mathbf{x})=-\sum_{c=0}^{j}\left(-x_{m}\right)^{-1-c} \sigma_{j-c}(\mathbf{x}) .
\end{aligned}
$$

From (3.19) we can easily find $S$ from (3.18):

$$
S_{i j}=N \operatorname{Res}_{z=0} L_{i j}^{\mathrm{top}}(z)=\sum_{m=1}^{N} \frac{\left(q_{m}+\eta\right)^{\varrho(i)} e^{p_{m} / c}}{\prod_{k \neq m}\left(q_{m}-q_{k}\right)}(-1)^{\varrho(j)} \sigma_{\varrho(j)}(\mathbf{q})
$$

To take into account the light speed $c$ in the quadratic brackets (2.20) one should put the common factor $1 / c$ in front of r.h.s. of (2.20). It is equivalent to redefinition of the classical $r$-matrix $r_{12}(z) \rightarrow r_{12}(z) / c$. 
Using (3.20)-(3.25) we can rewrite the Lax matrix (3.16) in terms of the variables $S$ (3.20). The computation gives:

$$
\begin{aligned}
& L^{\eta}(z)=N \tilde{L}^{\eta, c}(z, \mathbf{q}, \mathbf{p})=\frac{1}{z} \sum_{i, j=1}^{N} \mathrm{E}_{i j}\left\{\sum_{\gamma=0}^{\varrho(i)} z^{\gamma}\left(\begin{array}{c}
\varrho(i) \\
\gamma
\end{array}\right) S_{\varrho^{-1}(\varrho(i)-\gamma), j}\right. \\
& -\sum_{\gamma=0}^{\varrho(i)} z^{\gamma+N-j+1}(-1)^{\varrho(j)+N}(N-j)\left(\begin{array}{c}
\varrho(i) \\
\gamma
\end{array}\right)\left(\begin{array}{c}
N \\
j-1
\end{array}\right) S_{\varrho^{-1}(\varrho(i)-\gamma), N} \\
& +\sum_{\gamma=0}^{\varrho(i)} \sum_{s=1}^{N-j} z^{s+\gamma}(-1)^{\varrho(j)+s+j-1}\left(\begin{array}{c}
\varrho(i) \\
\gamma
\end{array}\right)\left(\begin{array}{c}
s+j-1 \\
j-1
\end{array}\right) S_{\varrho^{-1}(\varrho(i)-\gamma), \varrho^{-1}(s+j-1)}- \\
& -N \sum_{s=1}^{N-j} \sum_{b=0}^{\varrho(i)}(-1)^{\varrho(j)+s+j-1} z^{s}(z+\eta)^{b}\left(\begin{array}{c}
s+j-2 \\
j-1
\end{array}\right)\left(\begin{array}{c}
\varrho(i) \\
b
\end{array}\right) \times \\
& {\left[\delta_{\varrho(i)-j-s-b+1 \leq 0} \sum_{c=0}^{N-s-j+1} \sum_{p=0}^{\varrho(i)-b+c}(-\eta)^{p}\left(\begin{array}{c}
\varrho(i)-b+c \\
p
\end{array}\right) S_{\varrho^{-1}(\varrho(i)-b-p+c), \varrho^{-1}(s+j+c-1)}\right.} \\
& \left.\left.-\delta_{\varrho(i)-j-s-b+1>0} \sum_{c=0}^{s+j-2} \sum_{p=0}^{\varrho(i)-b-c-1}(-\eta)^{p}\left(\begin{array}{c}
\varrho(i)-b-c-1 \\
p
\end{array}\right) S_{\varrho^{-1}(\varrho(i)-b-p-c-1), \varrho^{-1}(s+j-c-2)}\right]\right\} \\
& +\frac{1}{z}\left[z N \mathrm{E}_{N N}-\sum_{j=1}^{N} z^{N-j+2}(-1)^{\varrho(j)+N}(N-j) N\left(\begin{array}{c}
N \\
j-1
\end{array}\right) \mathrm{E}_{N j}\right. \\
& -N \sum_{i, j=1}^{N} \sum_{s=1}^{N-j} \sum_{b=0}^{\varrho(i)}(-1)^{\varrho(j)+s+j-1} z^{s}(z+\eta)^{b}\left(\begin{array}{c}
s+j-2 \\
j-1
\end{array}\right)\left(\begin{array}{c}
\varrho(i) \\
b
\end{array}\right) \delta_{p \leq 1} \delta_{\varrho(i)-b-p-j-s+2,0} \times \\
& \left.\sum_{p=0}^{\varrho(i)-b+N-s-j+1}(-\eta)^{p}\left(\begin{array}{c}
\varrho(i)-b+N-s-j+1 \\
p
\end{array}\right) \mathrm{E}_{i j}\right] \times \\
& \times\left(-\frac{1}{N(-\eta)} \sum_{j=1}^{N} S_{j j}-\frac{1}{N^{2}} \sum_{j=1}^{N}\left[\delta_{\varrho(j) \geq 1} \varrho(j) S_{\varrho^{-1}(\varrho(j)-1), \varrho^{-1}(j)}+(-1)^{\varrho(j)+j} j S_{j, \varrho^{-1}(j)}\right.\right. \\
& \left.\left.-N \sum_{b=0}^{\varrho(j)}(-1)^{\varrho(j)+j+b}\left(\begin{array}{c}
\varrho(j) \\
b
\end{array}\right) \sum_{c=0}^{N-j} \sum_{p=0}^{\varrho(j)-b+c}(-\eta)^{p+b}\left(\begin{array}{c}
\varrho(j)-b+c \\
p
\end{array}\right) S_{\varrho^{-1}(\varrho(j)-b-p+c), \varrho^{-1}(j+c)}\right]\right)
\end{aligned}
$$

Here and below we imply that the values of indices corresponding to the undefined argument value $(N-1)$ of function $\varrho^{-1}(3.2)$ are skipped in the summations.

It is important to mention that the answer does not depend on the values of the Casimir functions (2.22). As we know it is defined by only quantum $R$-matrix which is the subject of the next section. Therefore, we can consider the obtained expression (3.27)-(3.30) as independent definition of the Lax matrix for the generic rational relativistic top. The case (3.16) which is gauge equivalent to the rational RS model appears for the particular 
values of the Casimir functions $C_{k}$ (2.22). From (3.17) we conclude that the RS case corresponds to:

$$
\text { RS : } \quad C_{-1}=\eta, \quad C_{0}=1, \quad C_{k}=0, k \neq-1,0 .
$$

In the non-relativistic limit this case corresponds to the rational top on the coadjoint orbit of minimal dimension $(2 N-2)$. In a general case the obtained model yields alternative description of the spin RS model [39].

The quantum Lax matrix is obtained from (3.27)-(3.30) by substitution $S \rightarrow \hat{S}$. In (3.26) it corresponds to $p_{j}:=\hbar \partial_{q_{j}}$ with the choice of normal ordering.

M-operator from non-relativistic limit. As it was shown in (2.33) the $M$-operator (2.29) coincides (up to minus) with the non-relativistic limit of the Lax matrix. Hence, we can use the answer obtained in $[6]:{ }^{8}$

$$
\begin{aligned}
& M_{i j}(z)=-\frac{1}{z} \times \\
& {\left[\sum_{\gamma=0}^{\varrho(i)} z^{\gamma}\left(\begin{array}{c}
\varrho(i) \\
\gamma
\end{array}\right) S_{\varrho^{-1}(\varrho(i)-\gamma), j}-\sum_{\gamma=0}^{\varrho(i)} z^{\gamma+N-j+1}(-1)^{\varrho(j)+N}(N-j)\left(\begin{array}{c}
\varrho(i) \\
\gamma
\end{array}\right)\left(\begin{array}{c}
N \\
j-1
\end{array}\right) S_{\varrho^{-1}(\varrho(i)-\gamma), N}\right.} \\
& -N \sum_{\gamma=0}^{\varrho(i)} \sum_{s=1}^{N-j} \delta_{\varrho(i)-j+1 \leq s+\gamma} z^{s+\gamma}(-1)^{\varrho(j)+s+j-1}\left(\begin{array}{c}
\varrho(i) \\
\gamma
\end{array}\right)\left(\begin{array}{c}
s+j-2 \\
j-1
\end{array}\right) \sum_{c=0}^{N-s-j+1} S_{\varrho^{-1}(\varrho(i)-\gamma+c), \varrho^{-1}(s+j+c-1)} \\
& -N \sum_{\gamma=0}^{\varrho(i)} \sum_{s=1}^{N-j} \delta_{\varrho(i)-j+1>s+\gamma} z^{s+\gamma}(-1)^{\varrho(j)+s+j-2}\left(\begin{array}{c}
\varrho(i) \\
\gamma
\end{array}\right)\left(\begin{array}{c}
s+j-2 \\
j-1
\end{array}\right) \sum_{c=0}^{s+j-2} S_{\varrho^{-1}(\varrho(i)-\gamma-c-1), \varrho^{-1}(s+j-c-2)} \\
& \left.\sum^{\varrho(i)} \sum_{\gamma=0}^{N-j} z^{s+\gamma}(-1)^{\varrho(j)+s+j-1}\left(\begin{array}{c}
\varrho(i) \\
\gamma
\end{array}\right)\left(\begin{array}{c}
s+j-1 \\
j-1
\end{array}\right) S_{\varrho^{-1}(\varrho(i)-\gamma), \varrho^{-1}(s+j-1)}-\frac{\delta_{i, j}}{N} \sum_{k=1}^{N} \sum_{c=0}^{N-k-2} S_{k+c, k+c+1}\right]
\end{aligned}
$$

Example: for $N=2(3.27)-(3.30)$ yields the Lax matrix

$$
L^{\eta}(z)=\frac{1}{z} S_{2 \times 2}+\frac{\operatorname{tr}(S)}{\eta} 1_{2 \times 2}-(z+\eta)\left(\begin{array}{cc}
S_{12} & 0 \\
\left(S_{11}-S_{22}\right)+\left(\eta^{2}+z^{2}+\eta z\right) S_{12}-S_{12}
\end{array}\right)
$$

with $S_{2 \times 2}=\left(\begin{array}{cc}S_{11} & S_{12} \\ S_{21} & S_{22}\end{array}\right)$ and $\operatorname{tr} S=S_{11}+S_{22}$. The determinant defines the Casimir functions

$$
\begin{aligned}
\operatorname{det} L^{\eta}(z) & =\frac{1}{z^{2}} C_{0}+\left(\frac{1}{z \eta}+\frac{1}{\eta^{2}}\right) C_{1}, \\
C_{0} & =\operatorname{det} S=S_{11} S_{22}-S_{12} S_{21}, \quad C_{1}=\left(S_{11}+S_{22}+\eta^{2} S_{12}\right)^{2}-4 \eta^{2} S_{12} S_{22}
\end{aligned}
$$

of the Poisson structure (2.20) for $\mathrm{gl}_{2}$. The Hamiltonian $S_{11}+S_{22}$ generates equations of motion (2.26) with the $M$-operator (2.29):

$$
M(z)=-\frac{1}{z}\left(\begin{array}{cc}
S_{11}-z^{2} S_{12} & S_{12} \\
S_{21}-z^{2}\left(S_{11}-S_{22}\right)-z^{4} S_{12} & S_{22}+z^{2} S_{12}
\end{array}\right)
$$

\footnotetext{
${ }^{8}$ Notice that in [6] the answer is $\mathrm{sl}_{N}$-valued. It differs from $(2.33)$ by scalar matrix and factor $-N$.
} 
In $N=2$ case $r_{12}^{(0)}$ from (2.13) vanishes. Hence, $J^{\eta}(S)(2.27)$ is defined by only $L^{\eta,(0)}$ from (2.1):

$$
J^{\eta}(S)=L^{\eta,(0)}=-\left(\begin{array}{cc}
\eta S_{12} & 0 \\
\eta^{3} S_{12}+\eta\left(S_{11}-S_{22}\right) & -\eta S_{12}
\end{array}\right)+\frac{S_{11}+S_{22}}{\eta} 1_{2 \times 2} .
$$

The last scalar term vanishes from the commutator in the equations $(2.26): \dot{S}=\left[S, J^{\eta}(S)\right]$.

\subsection{Spin chains and Gaudin models}

The Lax matrix (1.4), (3.27)-(3.30) allows to define a class of integrable $\mathrm{gl}_{N}$ spin chains with the transfer-matrix

$$
\hat{T}_{n}(z)=L^{\eta}\left(\hat{S}^{1}, z-z_{1}\right) \ldots L^{\eta}\left(\hat{S}^{n}, z-z_{n}\right) .
$$

on $n$ sites with inhomogenuities $z_{k}$. The underlying quantum algebra consists of $n$ copies of (2.7) with the quantum $R$-matrix (4.1)-(4.4). The non-relativistic limit gives rise to the Gaudin model defined by the Lax operator

$$
\hat{L}^{G}(z)=\sum_{a=1}^{n} l\left(z-z_{a}, \hat{S}^{a}\right) .
$$

Its Hamiltonians are computed as residues of $\operatorname{tr}\left(\hat{L}^{G}(z)\right)^{2}$ :

$$
\hat{h}_{a}=\sum_{c \neq a}^{n} h_{a, c}, \quad h_{a, c}=\operatorname{tr}_{12}\left(r_{12}\left(z_{a}-z_{c}\right) \hat{S}_{1}^{a} \hat{S}_{2}^{c}\right)=\operatorname{tr}\left(\hat{S}^{a} l\left(z_{a}-z_{c}, \hat{S}^{c}\right)\right) .
$$

For example, in $\mathrm{gl}_{2}$ case the classical $r$-matrix (2.13) (classical limit of (1.27))

$$
r_{12}(z)=\left(\begin{array}{cccc}
1 / z & 0 & 0 & 0 \\
-z & 0 & 1 / z & 0 \\
-z & 1 / z & 0 & 0 \\
-z^{3} & z & z & 1 / z
\end{array}\right)
$$

gives

$$
h_{a, c}=\frac{\operatorname{tr}\left(\hat{S}^{a} \hat{S}^{c}\right)}{z_{a}-z_{c}}-\left(z_{a}-z_{c}\right)\left(\hat{S}_{12}^{a}\left(\hat{S}_{11}^{c}-\hat{S}_{22}^{c}\right)+\hat{S}_{12}^{c}\left(\hat{S}_{11}^{a}-\hat{S}_{22}^{a}\right)\right)-\left(z_{a}-z_{c}\right)^{3} \hat{S}_{12}^{a} \hat{S}_{12}^{c} .
$$

In the limit (1.29) this formula reproduces the well-known rational Gaudin Hamiltonians (the first term in $(3.41)$ ).

Let us also compute the quantum local Hamiltonian of the homogeneous $\left(z_{a}=0\right)$ periodic spin chain on $n$ sites. The quantum $R$-matrix

$$
\tilde{R}^{\eta}(z)=z \eta R^{\eta}(z)
$$


with $R(1.27)$, satisfies

$$
\tilde{R}^{\eta}(0)_{12}=\eta P_{12} .
$$

Therefore, we can calculate the local Hamiltonian using standard approach of [15, 40-45]. The answer is given by $\sum_{k=1}^{n} H_{k, k+1}$, where $H_{k, k+1}=\left.P_{k, k+1} \frac{d}{d z} \tilde{R}_{k, k+1}^{\eta}(z)\right|_{z=0}$ :

$$
H^{\text {local }}=\sum_{k=1}^{n} P_{k, k+1}-\eta^{2} \mathrm{E}_{21}^{k} \otimes\left(\mathrm{E}_{11}^{k+1}-\mathrm{E}_{22}^{k+1}\right)-\eta^{2}\left(\mathrm{E}_{11}^{k}-\mathrm{E}_{22}^{k}\right) \otimes \mathrm{E}_{21}^{k+1}-\eta^{4} \mathrm{E}_{21}^{k} \otimes \mathrm{E}_{21}^{k+1},
$$

where $\mathrm{E}_{i j}^{n+1}=\mathrm{E}_{i j}^{1}$. It is a deformation of the XXX spin chain ${ }^{9}$. The latter is described by the only first term in (1.32):

$$
H^{\mathrm{xxx}}=\sum_{k=1}^{n} P_{k, k+1}, \quad P_{k, k+1}=\mathrm{E}_{11}^{k} \otimes \mathrm{E}_{11}^{k+1}+\mathrm{E}_{12}^{k} \otimes \mathrm{E}_{21}^{k+1}+\mathrm{E}_{21}^{k} \otimes \mathrm{E}_{12}^{k+1}+\mathrm{E}_{22}^{k} \otimes \mathrm{E}_{22}^{k+1} .
$$

The generators $\mathrm{E}_{i j},\left(\mathrm{E}_{i j}\right)_{a b}=\delta_{i a} \delta_{j b}$ are dual to $\hat{S}_{j i}=\operatorname{tr}\left(\mathrm{E}_{i j} \hat{S}\right)$.

We describe this type of models and related soliton equations in our next publication [54].

\section{Quantum rational $\mathrm{R}$-matrix}

The quantum non-dynamical $R$-matrix can be found by the standard procedure of the IRF-Vertex Correspondence starting from the rational RS model. Here we use another approach based on (1.9). Applying it to (3.27)-(3.30) we get

$$
\begin{aligned}
& R_{12}^{\hbar}(z)=\sum_{k, l=1}^{N} \frac{\partial L^{\hbar}(z)}{\partial S_{k l}} \otimes \mathrm{E}_{l k}=\frac{1}{z} \sum_{i, j=1}^{N} \mathrm{E}_{i j} \otimes\left\{\sum_{\gamma=0}^{\varrho(i)} z^{\gamma}\left(\begin{array}{c}
\varrho(i) \\
\gamma
\end{array}\right) \mathrm{E}_{j, \varrho^{-1}(\varrho(i)-\gamma)}\right. \\
& \quad-\sum_{\gamma=0}^{\varrho(i)} z^{\gamma+N-j+1}(-1)^{\varrho(j)+N}(N-j)\left(\begin{array}{c}
\varrho(i) \\
\gamma
\end{array}\right)\left(\begin{array}{c}
N \\
j-1
\end{array}\right) \mathrm{E}_{N, \varrho^{-1}(\varrho(i)-\gamma)} \\
& \quad+\sum_{\gamma=0}^{\varrho(i)} \sum_{s=1}^{N-j} z^{s+\gamma}(-1)^{\varrho(j)+s+j-1}\left(\begin{array}{c}
\varrho(i) \\
\gamma
\end{array}\right)\left(\begin{array}{c}
s+j-1 \\
j-1
\end{array}\right) \mathrm{E}_{\varrho^{-1}(s+j-1), \varrho^{-1}(\varrho(i)-\gamma)}- \\
& -N \sum_{s=1}^{N-j} \sum_{b=0}^{\varrho(i)}(-1)^{\varrho(j)+s+j-1} z^{s}(z+\hbar)^{b}\left(\begin{array}{c}
s+j-2 \\
j-1
\end{array}\right)\left(\begin{array}{c}
\varrho(i) \\
b
\end{array}\right) \times \\
& \left.\quad\left[\begin{array}{c}
\delta_{\varrho(i)-j-s-b+1 \leq 0} \sum_{c=0}^{N-s-j+1} \sum_{p=0}^{\varrho(i)-b+c}(-\hbar)^{p}\left(\begin{array}{c}
\varrho(i)-b+c \\
p
\end{array}\right) \mathrm{E}_{\varrho^{-1}(s+j+c-1), \varrho^{-1}(\varrho(i)-b-p+c)} \\
-\delta_{\varrho(i)-j-s-b+1>0} \sum_{c=0}^{s+j-2} \sum_{p=0}^{\varrho(i)-b-c-1}(-\hbar)^{p}\left(\begin{array}{c}
\varrho(i)-b-c-1 \\
p
\end{array}\right) \mathrm{E}_{\varrho^{-1}(s+j-c-2), \varrho^{-1}(\varrho(i)-b-p-c-1)}
\end{array}\right]\right\}
\end{aligned}
$$

\footnotetext{
${ }^{9}$ Another deformation of the Heisenberg chain was found in [46]. It is interesting to find some relation (if any) between two deformations.
} 


$$
\begin{aligned}
& +\frac{1}{z}\left[z N \mathrm{E}_{N N}-\sum_{j=1}^{N} z^{N-j+2}(-1)^{\varrho(j)+N}(N-j) N\left(\begin{array}{c}
N \\
j-1
\end{array}\right) \mathrm{E}_{N j}\right. \\
& -N \sum_{i, j=1}^{N} \sum_{s=1}^{N-j} \sum_{b=0}^{\varrho(i)}(-1)^{\varrho(j)+s+j-1} z^{s}(z+\hbar)^{b}\left(\begin{array}{c}
s+j-2 \\
j-1
\end{array}\right)\left(\begin{array}{c}
\varrho(i) \\
b
\end{array}\right) \delta_{p \leq 1} \delta_{\varrho(i)-b-p-j-s+2,0} \times \quad(4.3) \\
& \left.\quad \sum_{p=0}^{\varrho(i)-b+N-s-j+1}(-\hbar)^{p}\left(\begin{array}{c}
\varrho(i)-b+N-s-j+1 \\
p
\end{array}\right) \mathrm{E}_{i j}\right] \otimes \\
& \otimes\left(-\frac{1}{N(-\hbar)} \sum_{j=1}^{N} \mathrm{E}_{j j}-\frac{1}{N^{2}} \sum_{j=1}^{N}\left[\begin{array}{c}
\delta_{\varrho(j) \geq 1} \varrho(j) \mathrm{E}_{\varrho^{-1}(j), \varrho^{-1}(\varrho(j)-1)}+(-1)^{\varrho(j)+j} j \mathrm{E}_{\varrho^{-1}(j), j} \\
\left.\left.-N \sum_{b=0}^{\varrho(j)}(-1)^{\varrho(j)+j+b}\left(\begin{array}{c}
\varrho(j) \\
b
\end{array}\right) \sum_{c=0}^{N-j} \sum_{p=0}^{\varrho(j)-b+c}(-\hbar)^{p+b}\left(\begin{array}{c}
\varrho(j)-b+c \\
p
\end{array}\right) \mathrm{E}_{\varrho^{-1}(j+c), \varrho^{-1}(\varrho(j)-b-p+c)}\right]\right)
\end{array}\right.\right.
\end{aligned}
$$

As in (3.27)-(3.30) we imply that the values of indices corresponding to undefined argument value $N-1$ of $\varrho^{-1}$ function are skipped in summations. Notice that the obtained $R$-matrix is unitary (1.3) with $f^{\hbar}(z)=\frac{1}{\hbar^{2}}-\frac{1}{z^{2}}$ :

$$
R_{12}^{\hbar}(z) R_{21}^{\hbar}(-z)=\left(\frac{1}{\hbar^{2}}-\frac{1}{z^{2}}\right) 1 \otimes 1 .
$$

By redefinition

$$
R_{12}^{\hbar}(z, \epsilon)=\epsilon R_{12}^{\epsilon \hbar}(z \epsilon)
$$

we can treat the answer as deformation of the standard XXX $R$-matrix. Indeed, one can verify that

$$
\lim _{\epsilon \rightarrow 0} R_{12}^{\hbar}(z, \epsilon)=\hbar^{-1} 1 \otimes 1+z^{-1} P_{12} .
$$

In the classical limit we get the rational skew-symmetric non-dynamical $r$-matrix from $[6]:{ }^{10}$

$$
\begin{aligned}
& r^{\mathrm{top}}(z)=\frac{1}{N z} 1 \otimes 1+\frac{1}{z} \sum_{i, j=1}^{N} E_{i j} \otimes \\
& {\left[\sum_{\gamma=0}^{\varrho(i)} z^{\gamma}\left(\begin{array}{c}
\varrho(i) \\
\gamma
\end{array}\right) E_{\varrho^{-1}(\varrho(i)-\gamma), j}-\sum_{\gamma=0}^{\varrho(i)} z^{\gamma+N-j+1}(-1)^{\varrho(j)+N}(N-j)\left(\begin{array}{c}
\varrho(i) \\
\gamma
\end{array}\right)\left(\begin{array}{c}
N \\
j-1
\end{array}\right) E_{\varrho^{-1}(\varrho(i)-\gamma), N}\right.} \\
& -N \sum_{\gamma=0}^{\varrho} \sum_{s=1}^{N-j} \delta_{\varrho(i)-j+1 \leq s+\gamma} z^{s+\gamma}(-1)^{\varrho(j)+s+j-1}\left(\begin{array}{c}
\varrho(i) \\
\gamma
\end{array}\right)\left(\begin{array}{c}
s+j-2 \\
j-1
\end{array}\right) \sum_{c=0}^{N-s-j+1} E_{\varrho^{-1}(\varrho(i)-\gamma+c), \varrho^{-1}(s+j+c-1)} \\
& -N \sum_{\gamma=0}^{\varrho(i)} \sum_{s=1}^{N-j} \delta_{\varrho(i)-j+1>s+\gamma} z^{s+\gamma}(-1)^{\varrho(j)+s+j-2}\left(\begin{array}{c}
\varrho(i) \\
\gamma
\end{array}\right)\left(\begin{array}{c}
s+j-2 \\
j-1
\end{array}\right) \sum_{c=0}^{s+j-2} E_{\varrho^{-1}(\varrho(i)-\gamma-c-1), \varrho^{-1}(s+j-c-2)} \\
& \left.\quad+\sum_{\gamma=0}^{\varrho(i)} \sum_{s=1}^{N-j} z^{s+\gamma}(-1)^{\varrho(j)+s+j-1}\left(\begin{array}{c}
\varrho(i) \\
\gamma
\end{array}\right)\left(\begin{array}{c}
s+j-1 \\
j-1
\end{array}\right) E_{\varrho^{-1}(\varrho(i)-\gamma), \varrho^{-1}(s+j-1)}-\frac{\delta_{i, j}}{N} \sum_{k=1}^{N} \sum_{c=0}^{N-k-2} E_{k+c, k+c+1}\right]
\end{aligned}
$$

\footnotetext{
${ }^{10}$ Expression (4.8) differs from the one given in [6] by common factor $N$ and scalar term $1 \otimes 1 / N z$.
} 
Example: 11-vertex R-matrix. In $N=2$ case we obtain the 11-vertex R-matrix:

$$
R^{\hbar}(z)=\left(\begin{array}{cccc}
\hbar^{-1}+z^{-1} & 0 & 0 & 0 \\
-\hbar-z & \hbar^{-1} & z^{-1} & 0 \\
-\hbar-z & z^{-1} & \hbar^{-1} & 0 \\
-\hbar^{3}-2 z \hbar^{2}-2 \hbar z^{2}-z^{3} & \hbar+z \hbar+z \hbar^{-1}+z^{-1}
\end{array}\right)
$$

This $R$-matrix was obtained previously in [76] and later rediscovered in [74] by applying special limiting procedure to the Baxter-Belavin elliptic $R$-matrix. Author of [74] suggested the algorithm which allowed to get the answer using computer calculations. In this paper we obtain explicit answer for $\mathrm{gl}_{N}$ case using different approach. It is based on the relations to the classical RS model.

Example: rational $\mathrm{gl}_{\mathbf{3}} \boldsymbol{R}$-matrix. $\quad \mathrm{In}_{\mathrm{gl}}$ case $(4.1)-(4.4)$ gives the following $9 \times 9$ quantum $R$-matrix:

$$
\begin{aligned}
& R^{\hbar}(z)= \\
& \left(\begin{array}{ccc}
\hbar^{-1}+z^{-1} & 0 & 0 \\
1 & \hbar^{-1} & 0 \\
2 \hbar^{2}+3 z \hbar+2 z^{2} & -3 \hbar-3 z & \hbar^{-1} \\
-1 & z^{-1} & 0 \\
2 \hbar+2 z & 0 & 0 \\
2 z^{3}+3 z \hbar^{2}+2 \hbar^{3}+3 z^{2} \hbar & -3 \hbar^{2}-3 z \hbar-z^{2} & 1 \\
-2 \hbar^{2}-3 z \hbar-2 z^{2} & -3 \hbar-3 z & z^{-1} \\
2 z^{3}+3 z \hbar^{2}+2 \hbar^{3}+3 z^{2} \hbar & 3 z^{2}+3 z \hbar+\hbar^{2} & -1 \\
2 \hbar^{5}+3 z^{4} \hbar+3 z^{2} \hbar^{3}+2 z^{5}+3 z \hbar^{4}+3 z^{3} \hbar^{2} & 3 z^{4}-3 \hbar^{4}-3 z \hbar^{3}+3 z^{3} \hbar-z^{2}+\hbar^{2}
\end{array}\right. \\
& \begin{array}{llllll}
0 & 0 & 0 & 0 & 0 & 0
\end{array} \\
& \begin{array}{llllll}
z^{-1} & 0 & 0 & 0 & 0 & 0
\end{array} \\
& \begin{array}{llllll}
-3 \hbar-3 z & 3 & 0 & z^{-1} & 0 & 0
\end{array} \\
& \begin{array}{llllll}
\hbar^{-1} & 0 & 0 & 0 & 0 & 0
\end{array} \\
& \begin{array}{llllll}
0 & \hbar^{-1}+z^{-1} & 0 & 0 & 0 & 0
\end{array} \\
& \begin{array}{llllll}
-3 z \hbar-3 z^{2}-\hbar^{2} & 0 & \hbar^{-1} & 1 & z^{-1} & 0
\end{array} \\
& \begin{array}{llllll}
-3 \hbar-3 z & -3 & 0 & \hbar^{-1} & 0 & 0
\end{array}
\end{aligned}
$$

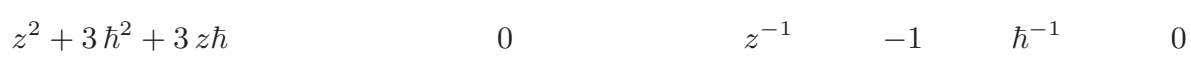

$$
\begin{aligned}
& \left.3 z \hbar^{3}+3 \hbar^{4}-3 z^{3} \hbar-3 z^{4}-6 \hbar^{3}-6 z^{3}-9 z \hbar^{2}-9 z^{2} \hbar 3 z+3 \hbar-\hbar^{2}+z^{2} 3 z+3 \hbar \hbar^{-1}+z^{-1}\right)
\end{aligned}
$$

Plugging it into (1.30) one gets gauge equivalent top-like form of 3-body rational RS model while for generic variables $S$ it can be considered as alternative description of the spin RS model. 


\section{Belavin's $\boldsymbol{R}$-matrix and elliptic models}

\subsection{Sklyanin algebra and relativistic elliptic tops}

Belavin's elliptic $\mathrm{gl}_{N} R$-matrix [16]

$$
R_{12}^{\hbar}(z)=\sum_{a \in \mathbb{Z}_{N} \times \mathbb{Z}_{N}} \varphi_{a}^{\hbar}(z) T_{a} \otimes T_{-a}
$$

is the central object of the section as well as related quantum $L$-operator:

$$
\hat{L}^{\eta}(z) \stackrel{(2.5)}{=} \operatorname{tr}_{2}\left(R_{12}^{\eta}(z) \hat{S}_{2}\right)=\sum_{a \in \mathbb{Z}_{N} \times \mathbb{Z}_{N}} \varphi_{a}^{\eta}(z) T_{a} \hat{S}_{a} .
$$

In (5.1) and (5.2) the basis of $\mathrm{gl}_{N}$ and corresponding functions are chosen as ${ }^{11}$

$$
\begin{gathered}
T_{a}=T_{a_{1} a_{2}}=\exp \left(\frac{\pi \imath}{N} a_{1} a_{2}\right) Q^{a_{1}} \Lambda^{a_{2}}, \quad Q_{k l}=\delta_{k l} \exp \left(\frac{2 \pi i}{N} k\right), \quad \Lambda_{k l}=\delta_{k-l+1=0 \bmod N}, \\
\varphi_{a}^{\eta}(z)=\exp \left(2 \pi \imath z \partial_{\tau} \omega_{a}\right) \phi\left(z, \omega_{a}+\eta\right), \quad \phi(z, u)=\frac{\vartheta^{\prime}(0) \vartheta(u+z)}{\vartheta(z) \vartheta(u)}, \quad \omega_{a}=\frac{a_{1}+a_{2} \tau}{N},
\end{gathered}
$$

where $\vartheta(z)$ is the odd theta function and $a_{1}, a_{2} \in \mathbb{Z}_{N}$.

The Sklyanin algebra (2.7) is defined by the local behavior of $\varphi_{a}^{\eta}(z)$ near $z=0$ :

$$
\phi(z, u)=\left(\frac{1}{z}+E_{1}(u)+\frac{z}{2}\left(E_{1}^{2}(u)-\wp(u)\right)+\ldots\right), \quad E_{1}(z)=\partial_{z} \log \vartheta(z) .
$$

Then

$$
R_{12}^{\hbar,(0)}(z)=\sum_{a \in \mathbb{Z}_{N} \times \mathbb{Z}_{N}} E_{1}\left(\omega_{a}+\hbar\right) T_{a} \otimes T_{-a}
$$

and

$$
\hat{L}^{\eta,(0)}(z)=\sum_{a \in \mathbb{Z}_{N} \times \mathbb{Z}_{N}}\left(E_{1}\left(\omega_{a}+\eta\right)+2 \pi \imath \partial_{\tau} \omega_{a}\right) T_{a} \hat{S}_{a} .
$$

The last terms $\left(2 \pi \imath \partial_{\tau} \omega_{a}\right)$ are canceled out in the final answers.

Sklyanin algebra $\mathcal{A}_{\hbar, \eta}^{\text {Skl }}$ for $\hbar, \eta \neq 0$ in components $\hat{S}_{a}=\operatorname{tr}\left(\hat{S} T_{-a}\right)$ can be derived from either (2.7) together with (5.6), (5.7) or directly - by plugging (5.1) and (5.2) into exchange relations (1.1). The latter way requires identity

$\varphi_{a-c}^{\eta}(z) \varphi_{b+c}^{\eta}(w) \varphi_{c}^{\hbar}(z-w)-\varphi_{b+c}^{\eta}(z) \varphi_{a-c}^{\eta}(w) \varphi_{a-b-c}^{\hbar}(z-w)=\mathbf{f}(a, b, c \mid \tau, \hbar, \eta) \varphi_{a}^{\eta+\hbar}(z) \varphi_{b}^{\eta-\hbar}(w)$, where

$$
\mathbf{f}(a, b, c \mid \tau, \hbar, \eta)=E_{1}\left(\omega_{c}+\hbar\right)-E_{1}\left(\omega_{a-b-c}+\hbar\right)+E_{1}\left(\omega_{a-c}+\eta\right)-E_{1}\left(\omega_{b+c}+\eta\right) .
$$

The quadratic relations $\mathcal{A}_{\tau, \hbar, \eta}^{\mathrm{Skl}}$ in the $T_{a} \otimes T_{b}$ component of (1.1) read as follows:

$$
\sum_{c \in \mathbb{Z}_{N} \times \mathbb{Z}_{N}} \mathbf{f}(a, b, c \mid \tau, \hbar, \eta)\left(\hat{S}_{a-c} \hat{S}_{b+c} \kappa_{c, a-b}-\hat{S}_{b+c} \hat{S}_{a-c} \kappa_{a-b, c}\right)=0,
$$

\footnotetext{
${ }^{11}$ See for example review [75].
} 
where $\kappa_{a, b}=\exp \frac{\pi \imath}{N}\left(a_{2} b_{1}-a_{1} b_{2}\right)$ comes from $T_{a} T_{b}=\kappa_{a, b} T_{a+b}$. Notice that in the case $\eta=\hbar$ and $b=0$ one should consider the limit $\eta \rightarrow \hbar$ in (5.8) which gives

$$
\begin{gathered}
\varphi_{a-c}^{\hbar}(z) \varphi_{b+c}^{\hbar}(w) \varphi_{c}^{\hbar}(z-w)-\varphi_{b+c}^{\hbar}(z) \varphi_{a-c}^{\hbar}(w) \varphi_{a-b-c}^{\hbar}(z-w)=\mathbf{f}(a, 0, c \mid \tau, \hbar, \hbar) \varphi_{a}^{2 \hbar}(z), \\
\mathbf{f}(a, 0, c \mid \tau, \hbar, \hbar)=E_{2}\left(\omega_{c}+\hbar\right)-E_{2}\left(\omega_{a}-\omega_{c}+\hbar\right) .
\end{gathered}
$$

The case $\eta=\hbar$ and $N=2$ in (5.9) gives rise to the Sklyanin algebra in its original form $[72,73]$.

It follows from (5.8) that the "structure constant" $\mathbf{f}(a, b, c \mid \tau \hbar, \eta)$ is double periodic with respect to the shifts

$$
\hbar \rightarrow \hbar+\mathbb{Z}+\mathbb{Z} \tau, \quad \eta \rightarrow \eta+\mathbb{Z}+\mathbb{Z} \tau
$$

Therefore, we can consider the pair $(\hbar, \eta)$ as points on two elliptic curves $\Sigma_{\tau}=\mathbb{C} /(\mathbb{Z}+\tau \mathbb{Z})$. Consider the upper half-plane $\mathcal{H}^{+} \subset \mathbb{C}$. The moduli space $\mathfrak{M}$ of elliptic curves is the result of the action of $\mathrm{SL}_{2}(\mathbb{Z})$ on $\mathcal{H}^{+}$by the Möbius transform

$$
\mathfrak{M}=\mathcal{H}^{+} / \mathrm{SL}_{2}(\mathbb{Z}), \quad\left(\tau \rightarrow \frac{\alpha \tau+\beta}{\gamma \tau+\delta}\right) .
$$

The modular transformations acts on the theta-function as

$$
\vartheta\left(\frac{v}{\alpha+\beta \tau} \mid \frac{\gamma+\delta \tau}{\alpha+\beta \tau}\right)=\zeta(\alpha+\beta \tau)^{\frac{1}{2}} \exp \left(\frac{\imath \pi \alpha v^{2}}{\alpha+\beta \tau}\right) \vartheta(v \mid \tau)
$$

where $\zeta^{8}=1$. Then we can find that $\mathbf{f}(a, b, c \mid \tau, \hbar, \eta)$ is modular invariant, and therefore, it is a well defined function on $\mathfrak{M}$. In this way the universal bundle

$$
\mathcal{E}_{\tau}=\begin{gathered}
\Sigma_{\tau} \times \Sigma_{\tau} \\
\downarrow \\
\mathfrak{M}
\end{gathered}
$$

plays the role of the moduli space of the algebra $\mathcal{A}_{\tau, \hbar, \eta}^{\text {Skl }}$. Then the moduli space is

$$
\operatorname{Mod}\left(\mathcal{A}_{\tau, \hbar, \eta}^{\mathrm{Skl}}\right)=\mathcal{E}_{\tau} .
$$

Relation between $\mathcal{A}_{\eta \neq 0}^{\mathrm{skl}}$ and $\mathcal{A}_{\eta=0}^{\mathrm{skl}}$. Following [23] ${ }^{12}$ let us clarify the relation between $\eta$-dependent and $\eta$-independent $L$-operators discussed above (2.58). Two descriptions are distinct from each other by quasiperiodic boundary conditions on the lattice $\mathbb{C} / \mathbb{Z}+\mathbb{Z} \tau$ :

$$
\begin{aligned}
L^{\eta}(z+1) & =Q^{-1} L^{\eta}(z) Q, & L^{\eta}(z+\tau) & =\exp (-2 \pi \imath \eta) \Lambda^{-1} L^{\eta}(z) \Lambda, \\
\mathrm{L}(z+1) & =Q^{-1} \mathrm{~L}(z) Q, & \mathrm{~L}(z+\tau) & =\Lambda^{-1} \mathrm{~L}(z) \Lambda,
\end{aligned}
$$

where $Q$ and $\Lambda$ are from (5.3). Notice that $\mathrm{L}(z)$ (5.14) is a section of $\operatorname{End} V$-bundle, where $V$ is the holomorphic vector bundle with the transition functions $Q$ and $\tilde{\Lambda}(z)=$

\footnotetext{
${ }^{12}$ In that paper $\eta=\hbar$ was considered.
} 
$\exp \left(2 \pi \imath\left(\frac{z}{N}+\frac{\tau}{2 N}\right)\right) \Lambda$. In the same time $L^{\eta}(z)$ should be considered as a map between $V$ and $V^{\prime}$, where $V^{\prime}$ is defined by the transition functions $Q$ and $\Lambda^{\prime}(z)=\exp (2 \pi \imath \eta) \Lambda(z)$.

Set $\eta_{0}=-\eta$ and $g^{\eta}(z)=1 / \phi(z-\eta, \eta)$ in (2.58). Then

$$
\frac{1}{\phi(z-\eta, \eta)} \hat{L}^{\eta}(z-\eta, \varphi(\hat{S}))=T_{0} \hat{S}_{0}+\sum_{a \neq 0} \varphi_{a}^{0}(z) T_{a} \hat{S}_{a}
$$

where

$$
\varphi(\hat{S})=T_{0} \hat{S}_{0}+\sum_{a \neq 0} \varphi_{a}^{0}(\eta) T_{a} \hat{S}_{a}
$$

i.e. the $\eta$-independent description is given by

$$
\mathrm{L}(z, \hat{S})=T_{0} \hat{S}_{0}+\sum_{a \neq 0} \varphi_{a}^{0}(z) T_{a} \hat{S}_{a}=T_{0} \hat{S}_{0}+\operatorname{tr}_{2}\left(r_{12}(z) \hat{S}\right)
$$

The underlying identity is very simple:

$$
\varphi_{a}^{\eta}(z-\eta) / \varphi_{0}^{\eta}(z-\eta)=\varphi_{a}^{0}(z) / \varphi_{a}^{0}(\eta) .
$$

The sum over $a \neq 0$ corresponds to $\operatorname{sl}_{N}$ part, i.e. $\hat{S}$ can be replaced with $\hat{\bar{S}}$ in the r.h.s. of (5.15) and (5.16) as in (2.57). The classical $r$-matrix (2.12) emerging in the $\eta$-independent form (2.57) is the Belavin-Drinfeld $\mathrm{sl}_{N} r$-matrix [17]:

$$
r_{12}(z)=\sum_{a \neq 0} \varphi_{a}^{0}(z) T_{a} \otimes T_{-a} .
$$

The scalar term $T_{0} \otimes T_{0} E_{1}(z)$ is not important here.

Relativistic top appears in the quasi-classical limit. The Lax matrix $(2.10)^{13}$

$$
L^{\eta}(z)=\operatorname{tr}_{2}\left(R_{12}^{\eta}(z) S_{2}\right)=\sum_{a \in \mathbb{Z}_{N} \times \mathbb{Z}_{N}} \varphi_{a}^{\eta}(z) T_{a} S_{a}
$$

together with $M$-matrix (2.29)

$$
M(z)=-\operatorname{tr}_{2}\left(r_{12}(z) S_{2}\right) \stackrel{(5.19)}{=}-\sum_{a \neq 0} \varphi_{a}^{0}(z) T_{a} S_{a}
$$

provides equations of motion (2.26) generated by the Hamiltonian $H=S_{0}$ :

$$
\partial_{t_{0}} S=\left\{S_{0}, S\right\}=\left[S, J^{\eta}(S)\right]
$$

where the inverse inertia tensor $J^{\eta}(2.27)$ :

$$
\begin{aligned}
J^{\eta}(S) & =\operatorname{tr}_{2}\left(\left(R_{12}^{\eta,(0)}-r_{12}^{(0)}\right) S_{2}\right)=T_{0} S_{0} E_{1}(\eta)+\sum_{a \neq 0} T_{a} S_{a}\left(E_{1}\left(\omega_{a}+\eta\right)-E_{1}\left(\omega_{a}\right)\right)= \\
& =S E_{1}(\eta)+\sum_{a \neq 0} T_{a} S_{a}\left(E_{1}\left(\omega_{a}+\eta\right)-E_{1}\left(\omega_{a}\right)-E_{1}(\eta)\right) .
\end{aligned}
$$

The first (scalar) term $T_{0} S_{0} E_{1}(\eta)$ in the upper line of (5.23) vanishes from the commutator in (5.22) as well as the first term $S E_{1}(\eta)$ in the lower line. To verify (5.22), (5.23) one needs the following identity:

$$
\varphi_{a}^{\eta}(z) \varphi_{b}^{0}(z)=\varphi_{a+b}^{\eta}(z)\left(E_{1}(z)+E_{1}\left(\omega_{a}+\eta\right)+E_{1}\left(\omega_{b}\right)-E_{1}\left(z+\omega_{a+b}+\eta\right)\right) .
$$

\footnotetext{
${ }^{13} \operatorname{In}(2.10) \mathcal{J}^{\eta}{ }_{a, b}(z)=\delta_{a+b} \varphi_{a}^{\eta}(z)$.
} 
Non-relativistic limit $\quad \eta \rightarrow 0$ coincides with $\eta$-independent description at the level of equations of motion because the Lax matrices (2.33) and (2.45) are the same up to the scalar term $S_{0} 1$. This is due to existence of bihamiltonian structure, i.e. any linear combination of the linear and quadratic Poisson brackets are again some Poisson bracket (see [37] for details).

The equations of motion (5.22) keep the same form in the limit with

$$
\mathrm{J}(S)=\lim _{\eta \rightarrow 0} \eta^{-1} J^{\eta}(\bar{S})=-\sum_{a \neq 0} T_{a} S_{a} E_{2}\left(\omega_{a}\right), \quad E_{2}(z)=-\partial_{z}^{2} \log \vartheta(z),
$$

where $\bar{S}$ is the $\operatorname{sl}_{N}$ part of $S$. The equations of motion $\dot{S}=[S, \mathrm{~J}(S)]$ are generated by the Lax pair $l(z)=\sum_{a \neq 0} \varphi_{a}^{0}(z) T_{a} S_{a}$ and the $M$-matrix (2.54):

$$
\mathcal{M}(z)=\sum_{a \neq 0} f_{a}(z) T_{a} S_{a}, \quad f_{a}(z)=\left.\partial_{\eta} \varphi_{a}^{\eta}(z)\right|_{\eta=0} .
$$

The underlying elliptic function identity is very well known [38]:

$$
\varphi_{a}^{0}(z) f_{b}(z)-\varphi_{b}^{0}(z) f_{a}(z)=\varphi_{a+b}^{0}(z)\left(E_{2}\left(\omega_{a}\right)-E_{2}\left(\omega_{b}\right)\right)=\varphi_{a+b}^{0}(z)\left(\wp\left(\omega_{a}\right)-\wp\left(\omega_{b}\right)\right) .
$$

This model was introduced in [47]. Its phase space is the coadjoint orbit of Lie group $\mathrm{GL}_{N}$. When dimension of the orbit is minimal $(2 N-2)$ the Lax matrix is gauge equivalent to the one of elliptic Calogero-Moser model. More detailed description can be found in [75]. Higher rank Sklyanin algebras in the context of integrable systems were also discussed in $[18,23,37]$.

\subsection{Poincaré invariance}

The Poincaré Lie algebra for the relativistic integrable systems [69, 70] is defined as

$$
\{\mathcal{H}, \mathcal{P}\}=0, \quad\{\mathcal{B}, \mathcal{H}\}=\mathcal{P}, \quad\{\mathcal{B}, \mathcal{P}\}=\mathcal{H} .
$$

The RS models can be obtained by symplectic (or the Poisson) reduction procedures from the cotangent bundles to a certain loop groups $[34,35] .{ }^{14}$ For the top-like (elliptic) Lax operators (1.19) a similar procedure was suggested in [18] and [23]. In all the description the Lax matrix appears through reduction from the group element. It satisfies some moment map constraint generated by the symmetries of the (co)adjoint action.

Let us show that the mentioned above reductions provide naturally the Poincaré Lie algebra (5.28). As a preliminary, consider the finite-dimensional case, i.e. the cotangent bundle $T^{*} G$ to $G=\mathrm{GL}_{N}$ Lie group. Let $g \in G$ and $A \in \mathrm{gl}_{N}^{*}$. The symplectic structure on $T^{*} G$ is equal to

$$
\omega=d \operatorname{tr}\left(A g^{-1} d g\right) .
$$

The corresponding Poisson brackets are of the form:

$$
\begin{aligned}
& \left\{A_{1}, A_{2}\right\}=\left[P_{12}, A_{2}\right], \quad\left\{g_{1}, g_{2}\right\}=0, \\
& \left\{A_{1}, g_{2}\right\}=g_{1} P_{12},
\end{aligned}
$$

\footnotetext{
${ }^{14}$ See also example of the relativistic Toda systems [71] in [31, 32].
} 
Taking $\operatorname{tr}_{1}$ of (5.31) we get

$$
\{\operatorname{tr} A, g\}=g .
$$

Therefore, $\left\{\operatorname{tr} A, g^{-1}\right\}=-g^{-1}$, and we have the following identification with (5.28):

$$
\mathcal{H}:=\operatorname{tr}\left(g+g^{-1}\right), \quad \mathcal{P}:=\operatorname{tr}\left(g-g^{-1}\right), \quad \mathcal{B}:=\operatorname{tr} A .
$$

Notice that the variable dual to the boost $\mathcal{B}$ is $\log (\operatorname{det} g)^{1 / N}$. Indeed, it follows from (5.32) that

$$
\{\operatorname{tr} A, \operatorname{det} g\}=N \operatorname{det} g .
$$

After reduction by the action of the gauge group

$$
g \rightarrow f^{-1} g f, \quad A \rightarrow f^{-1} A f, \quad f=f(A)
$$

the trivial Poisson brackets $\left\{g_{1}, g_{2}\right\}=0$ become those quadratic of the $r$-matrix form (1.18) [18]. The quantities $\mathcal{H}, \mathcal{P}$ and $\mathcal{B}$ from (5.33) are gauge invariants. The Poisson brackets (5.28) of the Poincaré Lie algebra keep the same form after reduction.

Similar reasoning is valid in more complicated cases. In [23] the classical Lax operator $\mathrm{L}(z, S)(5.17)$ (where $\hat{S} \rightarrow S$ ) was derived starting from the affine space over the cotangent bundle $T^{*} L L(G)$ to the two-loop group $L L(G), G=\mathrm{GL}_{N}$. Here we deal with the holomorphic bundle on the elliptic curve $\Sigma_{\tau}$ (with moduli $\tau$ ) given by transition functions $Q$ and $\tilde{\Lambda}=\exp \left(2 \pi \imath\left(-\frac{z}{N}-\frac{\tau}{2 N}\right)\right) \Lambda$ from (5.3). $\bar{A}-$ is the component of the $d_{\bar{A}}$ connection on $\Sigma_{\tau}$. In the Dolbeault description the holomorphic structure of the vector bundle is defined by the operator

$$
\begin{aligned}
d_{\bar{A}} & =\bar{\partial}+\bar{A}: \Omega^{(0,0)}\left(\Sigma_{\tau}, \operatorname{gl}(N, \mathbb{C})\right) \rightarrow \Omega^{(0,1)}\left(\Sigma_{\tau}, \operatorname{gl}(N, \mathbb{C})\right) \\
\bar{A}(z+1, \bar{z}+1) & =Q \bar{A}(z, \bar{z}) Q^{-1}, \quad \bar{A}(z+\tau, \bar{z}+\bar{\tau})=\Lambda \bar{A}(z, \bar{z}) \Lambda^{-1} .
\end{aligned}
$$

A section $\xi(z, \bar{z})$ is holomorphic if $d_{\bar{A}} \xi(z, \bar{z})=0$. Two holomorphic structures $d_{\bar{A}}$ and $d_{\bar{A}^{\prime}}$ are equivalent if they are related by the gauge transformation of the gauge group $\mathcal{G}$

$$
\bar{A} \rightarrow f^{-1} \bar{A} f+f^{-1} \bar{\partial} f .
$$

The quotient of the space of generic connections $\mathcal{A}=\left\{d_{\bar{A}}\right\}^{15}$ by the gauge group action is the moduli space $\operatorname{Bun}\left(\Sigma_{\tau}, \mathrm{GL}(N, \mathbb{C})\right)=\mathcal{A} / \mathcal{G}$ of holomorphic bundles. The initial phase space $\mathrm{P}_{\Sigma_{\tau}}$ is the Poisson algebra of holomorphic functionals on $\mathcal{R}$. The Poisson brackets are similar to $(5.30),(5.31)$ (see [23]). The gauge transformations (5.37) along with

$$
g \rightarrow f^{-1} g f, \quad f \in \mathcal{G}^{s} \subset \mathcal{G}:\left.\quad f(z, \bar{z})\right|_{z=0}=1
$$

leads to the finite-dimensional reduced phase space $\mathrm{P}^{\mathrm{red}}$. The reduced Poisson algebra coincides with (2.46) for the elliptic $r$-matrix (5.19), and

$$
\left.g(z, \bar{z})\right|_{\text {Pred }}=\mathrm{L}(z)=T_{0} S_{0}+\operatorname{tr}_{2}\left(r_{12}(z) S_{2}\right)=T_{0} S_{0}+\sum_{\alpha \neq 0} \varphi_{\alpha}^{0}(z) T_{\alpha} S_{\alpha} .
$$

\footnotetext{
${ }^{15}$ We don't discuss here the stability of the bundles, because we need only an open subset of $\mathcal{A}$.
} 
From (5.37) it follows that

$$
\operatorname{tr} \bar{A} \rightarrow \operatorname{tr} \bar{A}+\operatorname{tr}\left(f^{-1} \bar{\partial} f\right) .
$$

Since $\operatorname{tr} \bar{A}$ is double-periodic it can be gauge transformed to a constant $a$, i.e.

$$
\left.\operatorname{tr} \bar{A}(z, \bar{z})\right|_{\mathrm{Pred}}=a .
$$

Using the arguments similar to (5.30)-(5.32) one can see that

$$
\{a, \mathrm{~L}(z)\}=\mathrm{L}(z) .
$$

and reproduce the Poincaré algebra (5.28) in the form of (5.33) with $g=\mathrm{L}(z)$. The variable $a$ extends the phase space of the top. The variable dual to $a$ is the one which acts on the top variables by dilatation $S \rightarrow \lambda S$. This action does not preserves the values of the Casimir functions generated by $\operatorname{det} \mathrm{L}(z, S)$. Therefore, the Poincaré symmetry emerges on the top's phase space extended by the two-dimensional space $(a, \lambda)$ - cotangent bundle to the one-dimensional center of the group.

\subsection{Large $N$ limit: 2 d elliptic hydrodynamics}

In this paragraph we consider the large $N$ limit of the elliptic tops following and [37, 64] (see also [5]), where $\eta$-independent case was studied. This type of limit leads to $2 \mathrm{~d}$ hydrodynamics [8-10]. The idea is to replace the generators $(5.3)$ of $\mathrm{gl}_{N}$ with

$$
T_{a}:=\frac{i}{2 \pi \theta} \exp \left(2 \pi \imath \frac{a_{1} a_{2}}{2} \theta\right) U_{1}^{a_{1}} U_{2}^{a_{2}} \quad a \in \mathbb{Z}^{(2)}=\mathbb{Z} \oplus \mathbb{Z} .
$$

While $Q$ and $\Lambda$ from (5.3) gives the finite-dimensional representation of the Heisenberg group, the generators $U_{1}$ and $U_{2}$ satisfy commutation relation of the noncommutative torus $\mathcal{T}_{\theta}^{2}$ :

$$
U_{1} U_{2}=e^{-2 \pi i \theta} U_{2} U_{1}, \theta \in[0,1) .
$$

A generic element from $\mathcal{T}_{\theta}^{2}$ is $X=\sum_{a_{1}, a_{2} \in \mathbb{Z}} c_{a_{1}, a_{2}} U_{1}^{a_{1}} U_{2}^{a_{2}}, c_{a_{1}, a_{2}} \in \mathbb{C}$. This space is naturally identified with smooth functions on the two-dimensional torus $T^{2}=\left\{\mathbb{R}^{2} / \mathbb{Z} \oplus \mathbb{Z}\right\}$ :

$$
U_{1} \rightarrow \exp \left(2 \pi \imath x_{1}\right), U_{2} \rightarrow \exp \left(2 \pi \imath x_{2}\right), \quad 0<x_{1}, x_{2} \leq 1
$$

with the Moyal multiplication

$$
\exp \left(2 \pi \imath x_{1}\right) \star \exp \left(2 \pi \imath x_{2}\right)=e^{-2 \pi \imath \theta} \exp \left(2 \pi \imath x_{2}\right) \star \exp \left(2 \pi \imath x_{1}\right)
$$

or

$$
(f \star g)(x):=f g+\sum_{n=1}^{\infty} \frac{(\imath \pi \theta)^{n}}{(2 \pi \imath)^{2 n} n !} \varepsilon_{r_{1} p_{1}} \ldots \varepsilon_{r_{n} p_{n}}\left(\partial_{x_{r_{1}} \ldots x_{r_{n}}}^{n} f\right)\left(\partial_{x_{p_{1}} \ldots x_{p_{n}}}^{n} g\right)
$$

for functions

$$
f(x)=\sum_{a \in \mathbb{Z}^{(2)}} f_{a} T_{a}(x), \quad T_{a}(x)=\frac{\imath}{2 \pi \theta} \exp \left(2 \pi \imath \frac{a_{1} a_{2}}{2} \theta\right) \exp \left(2 \pi \imath a_{1} x_{1}\right) \exp \left(2 \pi \imath a_{2} x_{2}\right) .
$$


Then

$$
U_{1} f(x)=f(x-\theta), \quad U_{2} f(x)=\exp (2 \pi \imath x) f(x) .
$$

In other words, $U_{1}$ and $U_{2}$ are $\operatorname{GL}(\infty)$ analogues of $Q$ and $\Lambda$ from (5.3). The finitedimensional relations $T_{a} T_{b}=\kappa_{a, b} T_{a+b}$ are saved in the infinite-dimensional case with

$$
\kappa_{a, b}^{\theta}=-2 \pi \imath \theta \exp (\pi \imath \theta a \times b), \quad\left(a \times b=a_{2} b_{1}-a_{1} b_{2}\right) .
$$

Then we can introduce the following generalization of the Belavin's $R$-matrix (5.1) [37]:

$$
R_{12}^{\hbar}(z \mid \theta, \epsilon)=\sum_{a \in \mathbb{Z} \times \mathbb{Z}} \varphi_{a}^{\hbar}(z \mid \theta, \epsilon) T_{a} \otimes T_{-a}
$$

Here

$$
\begin{aligned}
\varphi_{a}^{\hbar}(z \mid \theta, \epsilon) & =\exp \left(2 \pi \imath \epsilon_{2} a_{2} z \theta\right) \phi\left(\left(\epsilon_{1} a_{1}+\tau \epsilon_{2} a_{2}\right) \theta+\hbar, z\right), \\
\epsilon & =\left(\epsilon_{1}, \epsilon_{2}\right), \quad \epsilon_{a} \theta<1 .
\end{aligned}
$$

and $T_{a}$ is the basis (5.43). It satisfies the Yang-Baxter equation (1.2). The related quantum $L$-operator is similar to (5.2):

$$
\hat{L}^{\eta}(z \mid \theta, \epsilon) \stackrel{(2.5)}{=} \operatorname{tr}_{2}\left(R_{12}^{\eta}(z) \hat{S}_{2}\right)=\sum_{a \in \mathbb{Z}_{N} \times \mathbb{Z}_{N}} \varphi_{a}^{\eta}(z \mid \theta, \epsilon) T_{a} \hat{S}_{a}
$$

where $\operatorname{tr}$ is defined as trace functional on $\mathcal{T}_{\theta}^{2}$ :

$$
\langle X\rangle=\operatorname{tr}(X)=c_{00},\langle 1\rangle=1, \quad\langle X Y\rangle=\langle Y X\rangle .
$$

In the Moyal representation: $\operatorname{tr} f=-\frac{1}{4 \pi^{2}} \int_{\mathcal{T}_{\theta}^{2}} f d x_{1} d x_{2}=f_{00}$.

The Lax operator (5.53) satisfies the quasi-periodicity conditions

$$
\left\{\begin{array}{l}
L^{\eta}(z+1)=U_{1}^{-\epsilon_{2}} L^{\eta}(z) U_{1}^{\epsilon_{2}}, \\
L^{\eta}(z+\tau)=\exp (2 \pi \imath \eta) U_{2}^{-\epsilon_{1}} L^{\eta}(z) U_{2}^{\epsilon_{1}},
\end{array}\right.
$$

Here $\epsilon_{1}$ and $\epsilon_{2}$ are arbitrary real numbers in the sense of (5.49). Conditions (5.55) mean that $L^{\eta}(z)$ is a section of the (twisted) Higgs bundle over elliptic curve $\Sigma_{\tau}$ with the structure group $S I N_{\theta}$. The latter consists of invertible elements of $\mathcal{T}_{\theta}^{2}$ (see e.g. [5]). The exchange relations (1.1) provide the direct generalization of the Sklyanin algebra (2.7):

$$
\begin{aligned}
& \mathcal{A}_{\tau, \hbar, \eta, \theta, \epsilon}^{\mathrm{Skl}}: \\
& P_{12} L^{\eta,(0)}(\theta, \epsilon)(\hat{S})_{1} \hat{S}_{2}+R_{12}^{\hbar,(0)}(\theta, \epsilon) \hat{S}_{1} \hat{S}_{2}=\hat{S}_{2} L^{\eta,(0)}(\theta, \epsilon)(\hat{S})_{1} P_{12}+\hat{S}_{2} \hat{S}_{1} R_{12}^{\hbar,(0)}(\theta, \epsilon) .
\end{aligned}
$$

with

$$
R_{12}^{\hbar,(0)}(z \mid \theta, \epsilon)=\sum_{a \in \mathbb{Z} \times \mathbb{Z}} E_{1}\left(\left(\epsilon_{1} a_{1}+\epsilon_{2} a_{2} \tau\right) \theta+\hbar\right) T_{a} \otimes T_{-a}
$$

and

$$
\hat{L}^{\eta,(0)}(z \mid \theta, \epsilon)=\sum_{a \in \mathbb{Z} \times \mathbb{Z}} E_{1}\left(\left(\epsilon_{1} a_{1}+\epsilon_{2} a_{2} \tau\right) \theta+\eta\right) T_{a} \hat{S}_{a} .
$$

Notice that this algebra depends on five parameters: $\hbar, \eta, \tau, \theta \epsilon_{1}, \theta \epsilon_{2}$. 
Classical limit $\hbar \rightarrow 0$ leads to $2 \mathrm{~d}$ elliptic hydrodynamics written in the Euler-Arnold form [8-10]. First, mention that the parameters $\epsilon_{1,2}$ (5.52) allow to define the complex structure on the noncommutative torus $\mathcal{T}_{\theta}^{2}$. For element $X=\sum_{a} c_{a} T_{a}$ define

$$
\bar{\partial}_{\epsilon, \tau} X=\sum_{a}\left(\epsilon_{1} a_{1}+\epsilon_{2} a_{2} \tau\right) c_{a} T_{a}
$$

The operator $J^{\eta}$ (5.23) acts as the following pseudo-differential operator:

$$
J^{\eta}(S)(x)=E_{1}\left(\eta+\theta \bar{\partial}_{\epsilon, \tau}\right) S(x)
$$

Consider analogue of the classical finite-dimensional Lax pair (5.20), (5.21):

$$
L^{\eta}(z, S(x))=\sum_{a \in \mathbb{Z}^{(2)}} S_{a} \varphi_{a}^{\eta}(z \mid \theta, \epsilon) T_{a}, \quad M(z, S(x))=\sum_{a \in \mathbb{Z}^{(2)}} S_{a} \varphi_{a}^{0}(z \mid \theta, \epsilon) T_{a} .
$$

Then the Lax equations provide equations of motion:

$$
\partial_{t} S(x)=\operatorname{ad}_{J^{\eta}(S)(x)}^{*} S(x)=\left[S(x), J^{\eta}(S)(x)\right]_{\theta},
$$

where

$$
[f(x), g(x)]_{\theta}=\theta^{-1}(f \star g-g \star f) .
$$

In components we have:

$$
\begin{aligned}
\partial_{t} S_{\alpha} & =\sum_{\gamma \in \mathbb{Z}^{(2)}} C_{\theta}(\alpha, \gamma) S_{\gamma} S_{\alpha-\gamma} E_{1}\left(\left(\epsilon_{1} \gamma_{1}+\epsilon_{2} \gamma_{2} \tau\right) \theta+\eta\right), \\
C_{\theta}(\alpha, \beta) & =\frac{1}{\pi \theta} \sin (\pi \theta(\alpha \times \beta)) .
\end{aligned}
$$

The obtained equations (5.62) can be treated as hydrodynamical limit of the elliptic spin Ruijsenaars-Schneider model. It is an interesting problem to find its relation to another type of hydrodynamical limit $[1,2]$. The latter approach leads to the quantum $\mathrm{gl}_{N}$ Benjamin-Ono and KdV systems while our approach gives rise to the $\mathrm{gl}_{N}$ Sklyanin type algebra (5.56) and classical equations (5.62), (5.63).

In the non-relativistic limit $\eta \rightarrow 0$ we reproduce the answer from [37,64]. Likewise the RS model goes into CM one, the relativistic top goes to non-relativistic (5.25). In the same way instead of $J^{\eta}$ (5.60) we get

$$
\mathrm{J}(S)(x)=-E_{2}\left(\theta \bar{\partial}_{\epsilon, \tau}\right) S(x) .
$$

In a similar way one can describe the dispersionless limit $\theta \rightarrow 0, \epsilon_{1,2} \rightarrow \infty$, $\lim _{\theta \rightarrow 0}\left(\theta \epsilon_{1,2}\right)=\epsilon_{1,2}^{\prime}<1$. In the limit the Lie algebra $\sin _{\theta}$ of the group $S I N_{\theta}$ becomes the Lie algebra $\operatorname{Ham}\left(T^{2}\right)$ of Hamiltonian vector fields on the two-dimensional torus. 


\section{Conclusion}

Let us briefly summarize the obtained results.

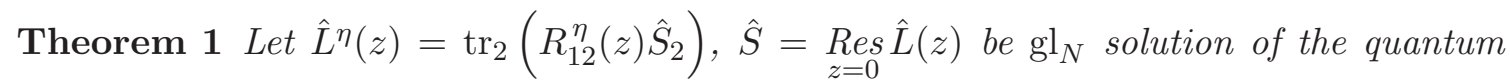
exchange relations (1.1) with the quantum non-dynamical $R$-matrix satisfying (1.2), (1.3) and (2.3), (2.12). Then

1) The quantum exchange relations (1.1) define the following $\mathrm{gl}_{N}$ Sklyanin algebra:

$$
\mathcal{A}_{\hbar, \eta}^{S k l}: \quad P_{12} L^{\eta,(0)}(\hat{S})_{1} \hat{S}_{2}+R_{12}^{\hbar,(0)} \hat{S}_{1} \hat{S}_{2}=\hat{S}_{2} L^{\eta,(0)}(\hat{S})_{1} P_{12}+\hat{S}_{2} \hat{S}_{1} R_{12}^{\hbar,(0)} .
$$

2) The $\mathrm{gl}_{N}$-valued Lax matrix

$$
L^{\eta}(z)=\operatorname{tr}_{2}\left(R_{12}^{\eta}(z) S_{2}\right), \quad S=\underset{z=0}{\operatorname{Res} L(z)}
$$

defines the classical integrable system described by the Poisson structure

$$
\begin{aligned}
\left\{L_{1}^{\eta}(z), L_{2}^{\eta}(w)\right\} & =\left[L_{1}^{\eta}(z) L_{2}^{\eta}(w), r_{12}(z-w)\right], \\
\mathcal{A}_{\hbar=0, \eta}^{S k l}: \quad\left\{S_{1}, S_{2}\right\} & =\left[S_{1} S_{2}, r_{12}^{(0)}\right]+\left[L^{\eta,(0)}(S)_{1} S_{2}, P_{12}\right] .
\end{aligned}
$$

3) The simplest Hamiltonian $\operatorname{tr}(S)$ generates top-like equations of motion

$$
\dot{S}=\left[S, J^{\eta}(S)\right]
$$

with the inverse inertia tensor

$$
J^{\eta}(S)=\operatorname{tr}_{2}\left(\left(R_{12}^{\eta,(0)}-r_{12}^{(0)}\right) S_{2}\right)
$$

where $R_{12}^{\eta,(0)}$ and $r_{12}^{(0)}$ are the coefficients of the local expansion of the quantum $R$ matrix (2.3) and the classical r-matrix (2.13) respectively.

4) Equations (6.4) are presented in the Lax form $\dot{L}^{\eta}(z)=\left[L^{\eta}(z), M(z)\right]$ with the $M$ operator given in terms of the classical r-matrix:

$$
M(z)=-\operatorname{tr}_{2}\left(r_{12}(z) S_{2}\right) .
$$

5) Alternatively, the relativistic top can be described in $\eta$-independent form as bihamiltonian system with the quadratic Poisson structure described in section 2.5 and the linear Poisson structure given in section 2.4. The relation between $\eta$-dependent and $\eta$-independent descriptions are given by (2.59).

Theorem 2 The classical Lax matrix (3.27)-(3.30) provides an example of relativistic integrable top described in Theorem 1. If $S$ is of rank one and $\operatorname{det} L^{\eta}(z)=\frac{z+\eta}{z}$ then the model is gauge equivalent to the rational $\mathrm{sl}_{N} R S$ model defined by the Lax matrix (3.4) with the change of variables

$$
S_{i j}(\mathbf{p}, \mathbf{q})=\sum_{m=1}^{N} \frac{\left(q_{m}+\eta\right)^{\varrho(i)} e^{p_{m} / c}}{\prod_{k \neq m}\left(q_{m}-q_{k}\right)}(-1)^{\varrho(j)} \sigma_{\varrho(j)}(\mathbf{q}) .
$$


Theorem 3 The quantum R-matrix (4.1)-(4.4) is a unitary solution of the Yang-Baxter equation (1.2) with $f^{\hbar}(z)=\hbar^{-2}-z^{-2}$ from (1.3). It can be obtained from the Lax matrix (3.27)-(3.30) as

$$
R_{12}^{\hbar}(z)=\sum_{k, l=1}^{N} \frac{\partial L^{\hbar}(z)}{\partial S_{k l}} \otimes \mathrm{E}_{l k} .
$$

In the limit (1.28)-(1.29) it gives the XXX R-matrix.

The latter statement can be obtained by the direct IRF-Vertex transformation starting from the quantum $R$-matrix for the rational RS model. We will give this proof elsewhere.

The obtained rational $R$-matrix allows to define new type of spin chains and Gaudin models. In the elliptic case, in addition to the relativistic top we describe the large $N$ limit as the elliptic hydrodynamics.

\section{Remarks}

- Relations between the Ruijsenaars-Schneider (RS) systems and quantum integrable chains appeared recently in the context of the Quantum-Classical duality using the Bethe ansatz approach [33] or the $\tau$-function approach [3, 4]. This duality, in particular, implies the substitution $\eta=\hbar$ into the Lax matrix of the RS model and provides an alternative (to the algebraic Bethe ansatz) method for computation of spectrum of the quantum spin chains transfer-matrices.

The phenomenon of the Quantum-Classical duality type was also observed at the level of gauge theories in the series of papers [58-62]. In this approach the Planck constant (in quantum integrable system) was identified with the twisted mass parameter in the $\mathcal{N}=2^{*}$ SUSY Yang-Mills theory. The latter mass parameter is related to the action of the global $\mathrm{U}(1)$ group on the adjoint chiral multiplet field. It resembles the appearance of the $\eta$ parameter in the twisted boundary conditions for the Lax operator (5.13), i.e. the twisted mass and the $\eta$ play similar roles and can be closely related.

A relation between the classical and quantum systems arises also in studies of the spectral duality [55-57]. A general statement is that the spectral duality works in the same way both at classical and quantum levels, or the properly defined classical and quantum spectral curves for spin chains coincide. It also resembles the similarity of the quantum $R$-matrices and the classical Lax operators.

- Expression (4.1)-(4.4) for the rational $R$-matrix is complicated. In the same time, the computations of particular examples emerge a lot of cancellations. We hope that the answer can be simplified. It is an interesting problem to find some elegant form for the rational $R$-matrix of group-theoretical type.

- The classification of integrable systems of Hitchin type on elliptic curves can be naturally made in terms of characteristic classes of underlying Higgs bundles [48-51, 75]. In particular, it allows one to obtain intermediate solutions of the quantum YangBaxter equation (between pure dynamical for RS model and pure non-dynamical 
one) $[52,53]$. In the rational case we deal with degenerated (and punctured) elliptic curve $y^{2}=z^{3}$. It is interesting to know whether elliptic classification survives in the rational limit.

- In this paper we do not consider the trigonometric case. However, it would appear reasonable that the obtained results are valid in this case as well. The corresponding non-dynamical quantum $R$-matrix was obtained in [7] from the trigonometric RS model via the IRF-Vertex transformation. It can be used for construction of trigonometric top-like models.

Open Access. This article is distributed under the terms of the Creative Commons Attribution License (CC-BY 4.0), which permits any use, distribution and reproduction in any medium, provided the original author(s) and source are credited.

\section{References}

[1] A.G. Abanov and P.B. Wiegmann, Quantum hydrodynamics, quantum Benjamin-Ono equation and Calogero model, Phys. Rev. Lett. 95 (2005) 076402 [cond-mat/0504041] [INSPIRE].

[2] G. Bonelli, A. Sciarappa, A. Tanzini and P. Vasko, Six-dimensional supersymmetric gauge theories, quantum cohomology of instanton moduli spaces and $g l(N)$ Quantum Intermediate Long Wave Hydrodynamics, arXiv:1403.6454 [INSPIRE].

[3] A. Alexandrov, S. Leurent, Z. Tsuboi and A. Zabrodin, The master T-operator for the Gaudin model and the KP hierarchy, Nucl. Phys. B 883 (2014) 173 [arXiv:1306.1111] [INSPIRE].

[4] A. Zabrodin, The Master T-Operator for Inhomogeneous XXX Spin Chain and mKP Hierarchy, SIGMA 10 (2014) 006 [arXiv:1310.6988] [INSPIRE].

[5] G. Aminov, S. Arthamonov, A.M. Levin, M.A. Olshanetsky and A.V. Zotov, Painleve Field Theory, arXiv:1306.3265 [INSPIRE].

[6] G. Aminov, S. Arthamonov, A.V. Smirnov and A.V. Zotov, Rational Top and its Classical R-matrix, arXiv:1402.3189 [INSPIRE].

[7] A. Antonov, K. Hasegawa and A. Zabrodin, On trigonometric intertwining vectors and nondynamical $R$ matrix for the Ruijsenaars model, Nucl. Phys. B 503 (1997) 747 [hep-th/9704074] [INSPIRE].

[8] V.I. Arnold, Sur la gomtrie diffrentielle des groupes de Lie de dimension infinie et ses applications l'hydrodynamique des fluides parfaits, Ann. Inst. Fourier 16 (1966) 316 [http://www.ams.org/mathscinet-getitem?mr=0202082].

[9] V.I. Arnold, The Hamiltonian nature of the Euler equations in the dynamics of a rigid body and of an ideal fluid, Uspekhi Mat. Nauk. 24 (1969) 225 [http://www.ams.org/mathscinet-getitem? $\mathrm{mr}=277163]$.

[10] V.I. Arnold and B.A. Khesin, Topological methods in hydrodynamics, Springer (1998).

[11] G.E. Arutyunov and S.A. Frolov, On Hamiltonian structure of the spin Ruijsenaars-Schneider model, J. Phys. A 31 (1998) 4203 [hep-th/9703119] [InSPIRE]. 
[12] J. Avan and G. Rollet, The Classical r matrix for the relativistic Ruijsenaars-Schneider system, Phys. Lett. 212 (1996) 50 [hep-th/9510166] [INSPIRE].

[13] F.W. Nijhoff, V.B. Kuznetsov, E.K. Sklyanin and O. Ragnisco, Dynamical $r$ matrix for the elliptic Ruijsenaars-Schneider system, J. Phys. A 29 (1996) L333 [solv-int/9603006] [INSPIRE].

[14] O. Babelon and C.M. Viallet, Hamiltonian Structures and Lax Equations, Phys. Lett. B 237 (1990) 411 [inSPIRE].

[15] R.J. Baxter, Partition function of the eight vertex lattice model, Annals Phys. 70 (1972) 193 [INSPIRE].

[16] A.A. Belavin, Dynamical Symmetry of Integrable Quantum Systems, Nucl. Phys. B 180 (1981) 189 [InSPIRE].

[17] A.A. Belavin and V.G. Drinfeld, Solutions of the classical Yang-Baxter equation for simple Lie algebras, Funct. Anal. Appl. 16 (1982) 159.

[18] H.W. Braden, V.A. Dolgushev, M.A. Olshanetsky and A.V. Zotov, Classical R matrices and the Feigin-Odesskii algebra via Hamiltonian and Poisson reductions,

J. Phys. A 36 (2003) 6979 [hep-th/0301121] [INSPIRE].

[19] F. Calogero, Exactly Solvable One-Dimensional Many Body Problems, Lett. Nuovo Cim. 13 (1975) 411 [INSPIRE].

[20] F. Calogero, On a Functional Equation Connected with Integrable Many Body Problems, Lett. Nuovo Cim. 16 (1976) 77 [INSPIRE].

[21] J. Moser, Three integrable Hamiltonian systems connected with isospectral deformations, Adv. Math. 16 (1975) 1.

[22] M.A. Olshanetsky and A.M. Perelomov, Classical integrable finite dimensional systems related to Lie algebras, Phys. Rept. 71 (1981) 313 [INSPIRE].

[23] Yu. Chernyakov, A. Levin, M. Olshanetsky and A. Zotov, Quadratic algebras related to elliptic curves, Theor. Math. Phys. 156 (2008) 1103 [arXiv:0710.1072.]

[24] L. De Broglie, D. Bohm, P. Hillion, F. Halbwachs, T. Takabayasi and J.-P. Vigier, Rotator model of elementary particles considered as relativistic extended structures in Minkowski space. Phys. Rev. 129 (1962) 438.

[25] A.J. Hanson and T. Regge, The Relativistic Spherical Top, Annals Phys. 87 (1974) 498 [INSPIRE].

[26] T. Takabayasi, Theory of relativistic top and electromagnetic interaction, Prog. Theor. Phys. 67 (1982) 357 [INSPIRE].

[27] R. Endelman and T.J. Hodges, Generalized Jordanian R-matrices of Cremmer-Gervais type, Lett. Math. Phys. 52 (2000) 225.

[28] R. Endelman and T.J. Hodges, Quantization of certain skew-symmetric solutions of the classical Yang-Baxter equation, math.QA/0003066.

[29] R. Endelman and T.J. Hodges, Degenerations and Representations of Twisted Shibukawa-Ueno R-Operators, Lett. Math. Phys. 68 (2004) 151 [math.QA/0402319].

[30] L. Fehér and B.G. Pusztai, The non-dynamical r-matrices of the degenerate Calogero-Moser models, J. Phys. A 33 (2000) 7739 [math-ph/0005021]. 
[31] V.V. Fock and A. Marshakov, A note on quantum qroups and relativistic toda theory, Nucl. Phys. B Proc. Suppl. 56 (1997) 208.

[32] A. Marshakov, Lie Groups, Cluster Variables and Integrable Systems, J. Geom. Phys. 67 (2013) 16 [arXiv:1207.1869] [INSPIRE].

[33] A. Gorsky, A. Zabrodin and A.V. Zotov, Spectrum of Quantum Transfer Matrices via Classical Many-Body Systems, JHEP 01 (2014) 070 [arXiv:1310.6958] [INSPIRE].

[34] A. Gorsky and N. Nekrasov, Relativistic Calogero-Moser model as gauged WZW theory, Nucl. Phys. B 436 (1995) 582 [hep-th/9401017] [INSPIRE].

[35] G.E. Arutyunov, S.A. Frolov and P.B. Medvedev, Elliptic Ruijsenaars-Schneider model from the cotangent bundle over the two-dimensional current group, J. Math. Phys. 38 (1997) 5682 [hep-th/9608013] [INSPIRE].

[36] K. Hasegawa, Ruijsenaars' commuting difference operators as commuting transfer matrices, Commun. Math. Phys. 187 (1997) 289 [q-alg/9512029].

[37] B. Khesin, A.M. Levin and M.A. Olshanetsky, Bihamiltonian structures and quadratic algebras in hydrodynamics and on non-commutative torus, Commun. Math. Phys. 250 (2004) 581 [nlin/0309017].

[38] I. Krichever, Elliptic solutions of the Kadomtsev-Petviashvili equation and integrable systems of particles, Funct. Anal. Appl. 14 (1980) 282.

[39] I. Krichever and A. Zabrodin, Spin generalization of the Ruijsenaars-Schneider model, nonAbelian $2-D$ Toda chain and representations of Sklyanin algebra, Russ. Math. Surv. 50 (1995) 1101 [hep-th/9505039] [INSPIRE].

[40] P.P. Kulish and E.K. Sklyanin, Quantum inverse scattering method and the Heisenberg ferromagnet, Phys. Lett. A 70 (1979) 461 [INSPIRE].

[41] L.D. Faddeev, E.K. Sklyanin and L.A. Takhtajan, The Quantum Inverse Problem Method. 1, Theor. Math. Phys. 40 (1980) 688 [inSPIRE].

[42] L.D. Faddeev, How algebraic Bethe ansatz works for integrable model, proceedings of Les-Houches summer school 64, A. Connes, K. Gawedzki and J. Zinn-Justin eds., North Holland (1998) [hep-th/9605187] [INSPIRE].

[43] V.E. Korepin, N.M. Bogoliubov and A.G. Izergin, Quantum Inverse Scattering Method and Correlation Functions, Cambridge Monographs on Mathematical Physics (1997).

[44] M. Gaudin, La Fonction d'Onde de Bethe, Masson (1983), Paris, (in French) Mir, (1987) Moscow (Russian transl.)

[45] N.A. Slavnov, The algebraic Bethe ansatz and quantum integrable systems, http://dx.doi.org/10.1070/RM200\%v062n04ABEH004430Russ. Math. Surv. 62 (2007) 727.

[46] P.P. Kulish and A.A. Stolin, Deformed Yangians and Integrable Models, Czech. Jour. Phys. 47 (1997) 1207 [q-alg/9708024].

[47] A.M. Levin, M.A. Olshanetsky and A.V. Zotov, Hitchin systems, symplectic Hecke correspondence and two-dimensional version, Commun. Math. Phys. 236 (2003) 93 [nlin/0110045].

[48] A.M. Levin, M.A. Olshanetsky, A.V. Smirnov and A.V. Zotov, Characteristic Classes and Integrable Systems. General Construction, arXiv:1006.0702 [INSPIRE]. 
[49] A.M. Levin, M.A. Olshanetsky, A.V. Smirnov and A.V. Zotov, Characteristic Classes and Integrable Systems for Simple Lie Groups, arXiv:1007.4127 [INSPIRE].

[50] A.M. Levin, M.A. Olshanetsky, A.V. Smirnov and A.V. Zotov, Hecke Transformations of Conformal Blocks in WZW Theory. I. KZB Equations for Non-trivial Bundles, SIGMA 8 (2012) 095 [arXiv: 1207.4386] [INSPIRE].

[51] A.M. Levin, M.A. Olshanetsky and A.V. Zotov, Classification of Isomonodromy Problems on Elliptic Curves, arXiv:1311.4498 [INSPIRE].

[52] A.M. Levin, M.A. Olshanetsky, A.V. Smirnov and A.V. Zotov, Characteristic Classes of $S L(N, \mathbb{C})$-Bundles and Quantum Dynamical Elliptic R-Matrices,

J. Phys. A 46 (2013) 035201 [arXiv:1208.5750] [inSPIRE].

[53] A.M. Levin and, A.V. Zotov, Integrable model of interacting elliptic tops, Theor. Math. Phys. $146(2006) 55$.

[54] A.M. Levin, M.A. Olshanetsky and A.V. Zotov, Classical Integrable Systems and Soliton Equations related to Eleven-Vertex R-Matrix, in preparation (2014).

[55] A. Mironov, A. Morozov, Y. Zenkevich and A.V. Zotov, Spectral Duality in Integrable Systems from AGT Conjecture, JETP Lett. 97 (2013) 45 [arXiv:1204.0913] [INSPIRE].

[56] A. Mironov, A. Morozov, B. Runov, Y. Zenkevich and A.V. Zotov, Spectral Duality Between Heisenberg Chain and Gaudin Model, Lett. Math. Phys. 103 (2013) 299 [arXiv:1206.6349] [INSPIRE].

[57] A. Mironov, A. Morozov, B. Runov, Y. Zenkevich and A.V. Zotov, Spectral dualities in XXZ spin chains and five dimensional gauge theories, JHEP 12 (2013) 034 [arXiv:1307.1502] [INSPIRE].

[58] N.A. Nekrasov and S.L. Shatashvili, Quantum integrability and supersymmetric vacua, Prog. Theor. Phys. Suppl. 177 (2009) 105 [arXiv:0901.4748] [InSPIRE].

[59] N.A. Nekrasov and S.L. Shatashvili, Supersymmetric vacua and Bethe ansatz, Nucl. Phys. Proc. Suppl. 192-193 (2009) 91 [arXiv:0901.4744] [INSPIRE].

[60] N.A. Nekrasov and S.L. Shatashvili, Quantization of Integrable Systems and Four Dimensional Gauge Theories, arXiv:0908.4052 [INSPIRE].

[61] N. Nekrasov, A. Rosly and S. Shatashvili, Darboux coordinates, Yang-Yang functional and gauge theory, Nucl. Phys. Proc. Suppl. 216 (2011) 69 [arXiv:1103.3919] [InSPIRE].

[62] N.A. Nekrasov and S.L. Shatashvili, Bethe/Gauge correspondence on curved spaces, arXiv: 1405.6046 [INSPIRE].

[63] A.V. Odesskii and B.L. Feigin, Sklyanin elliptic algebras, Funct. Anal. Appl. 23 (1989) 207.

[64] M.A. Olshanetsky, Elliptic hydrodynamics and quadratic algebras of vector fields on a torus, Theor. Math. Phys. 150 (2007) 301.

[65] V. Pasquier, Etiology of IRF Models, Commun. Math. Phys. 118 (1988) 355 [inSPIRE].

[66] M. Jimbo, T. Miwa and M. Okado, Local state probabilities of solvable lattice models: An $A_{n}^{(1)}$ family, Nucl. Phys. B 300 (1988) 74.

[67] E. Rains, S. Ruijsenaars, Difference operators of Sklyanin and van Diejen type, Commun. Math. Phys. 320 (2013) 851 [arXiv:1203.0042]. 
[68] H. Rosengren, Sklyanin invariant integration, Intern. Math. Res. Not. 60 (2004) 3207 [math.QA/0405072].

[69] S.N.M. Ruijsenaars, The Aharonov-Bohm Effect and Scattering Theory, Annals Phys. 146 (1983) 1 [inSPIRE].

[70] S.N.M. Ruijsenaars, Complete Integrability of Relativistic Calogero-moser Systems and Elliptic Function Identities, Commun. Math. Phys. 110 (1987) 191 [InSPIRE].

[71] S.N.M. Ruijsenaars, Relativistic Toda systems, Commun. Math. Phys. 133 (1990) 217.

[72] E.K. Sklyanin, Some algebraic structures connected with the Yang-Baxter equation, Funct. Anal. Appl. 16 (1982) 263 [INSPIRE].

[73] E.K. Sklyanin, Some algebraic structures connected with the Yang-Baxter equation. Representations of quantum algebras, Funct. Anal. Appl. 17 (1983) 273 [INSPIRE].

[74] A.V. Smirnov, Degenerate Sklyanin algebras, Cent. Eur. J. Phys. 8 (2010) 542 [arXiv:0903.1466].

[75] A.V. Smirnov and A.V. Zotov, Modifications of Bundles, Elliptic Integrable Systems and Related Problems, Theor. Math. Phys. 177 (2013) 1281.

[76] I.V. Cherednik, On a method of constructing factorized $S$ matrices in elementary functions, Theor. Math. Phys. 43 (1980) 356. 